\title{
$k$-Nearest Neighbor Classification over Semantically Secure Encrypted Relational Data
}

\author{
Bharath K. Samanthula, Yousef Elmehdwi and Wei Jiang \\ Email: \{bspq8, ymez76, wjiang\}@mst.edu
}

March 10, 2014

Technical Report

Department of Computer Science, Missouri S\&T

500 West 15th Street, Rolla, Missouri 65409 


\begin{abstract}
Data Mining has wide applications in many areas such as banking, medicine, scientific research and among government agencies. Classification is one of the commonly used tasks in data mining applications. For the past decade, due to the rise of various privacy issues, many theoretical and practical solutions to the classification problem have been proposed under different security models. However, with the recent popularity of cloud computing, users now have the opportunity to outsource their data, in encrypted form, as well as the data mining tasks to the cloud. Since the data on the cloud is in encrypted form, existing privacy preserving classification techniques are not applicable. In this paper, we focus on solving the classification problem over encrypted data. In particular, we propose a secure $k$-NN classifier over encrypted data in the cloud. The proposed $k$-NN protocol protects the confidentiality of the data, user's input query, and data access patterns. To the best of our knowledge, our work is the first to develop a secure $k$-NN classifier over encrypted data under the standard semi-honest model. Also, we empirically analyze the efficiency of our solution through various experiments.
\end{abstract}

Keywords - Security, $k$-NN Classifier, Outsourced Databases, Encryption

\title{
1 Introduction
}

Recently, the cloud computing paradigm [10, 42] is revolutionizing the organizations' way of operating their data particularly in the way they store, access and process data. As an emerging computing paradigm, cloud computing attracts many organizations to consider seriously regarding cloud potential in terms of its cost-efficiency, flexibility, and offload of administrative overhead. Most often, the organizations delegate their computational operations in addition to their data to the cloud.

Despite tremendous advantages that the cloud offers, privacy and security issues in the cloud are preventing companies to utilize those advantages. When data are highly sensitive, the data need to be encrypted before outsourcing to the cloud. However, when data are encrypted, irrespective of the underlying encryption scheme, performing any data mining tasks becomes very challenging without ever decrypting the data [46, 49]. In addition, there are other privacy concerns, demonstrated by the following example.

Example 1 Suppose an insurance company outsourced its encrypted customers database and relevant data mining tasks to a cloud. When an agent from the company wants to determine the risk level of a potential new customer, the agent can use a classification method to determine the risk level of the customer. First, the agent needs to generate a data record q for the customer containing certain personal information of the customer, e.g., credit score, age, marital status, etc. Then this record can be sent to the cloud, and the cloud will compute the class label for q. Nevertheless, since q contains sensitive information, to protect the customer's privacy, q should be encrypted before sending it to the cloud.

The above example shows that data mining over encrypted data (DMED) on a cloud also needs to protect a user's record when the record is a part of a data mining process. Moreover, cloud can also derive useful and sensitive information about the actual data items by observing the data access patterns even if the data are encrypted [19, 52]. Therefore, the privacy/security requirements of the DMED problem on a cloud are threefold: (1) confidentiality of the encrypted data, (2) confidentiality of a user's query record, and (3) hiding data access patterns.

Existing work on Privacy-Preserving Data Mining (either perturbation or secure multi-party computation based approach) cannot solve the DMED problem. Perturbed data do not possess semantic security, so data perturbation techniques cannot be used to encrypt highly sensitive data. Also the perturbed data do not produce very accurate data mining results. Secure multi-party computation based approach assumes data are distributed and not encrypted at each participating party. In addition, many intermediate computations are performed based on non-encrypted data. 
As a result, in this paper, we proposed novel methods to effectively solve the DMED problem assuming that the encrypted data are outsourced to a cloud. Specifically, we focus on the classification problem since it is one of the most common data mining tasks. Because each classification technique has their own advantage, to be concrete, this paper concentrates on executing the $k$-nearest neighbor classification method over encrypted data in the cloud computing environment.

\subsection{Problem Definition}

Suppose Alice owns a database $D$ of $n$ records $t_{1}, \ldots, t_{n}$ and $m+1$ attributes. Let $t_{i, j}$ denote the $j^{\text {th }}$ attribute value of record $t_{i}$. Initially, Alice encrypts her database attribute-wise, that is, she computes $E_{p k}\left(t_{i, j}\right)$, for $1 \leq i \leq n$ and $1 \leq j \leq m+1$, where column $(m+1)$ contains the class labels. We assume that the underlying encryption scheme is semantically secure [45]. Let the encrypted database be denoted by $D^{\prime}$. We assume that Alice outsources $D^{\prime}$ as well as the future classification process to the cloud.

Let Bob be an authorized user who wants to classify his input record $q=\left\langle q_{1}, \ldots, q_{m}\right\rangle$ by applying the $k$-NN classification method based on $D^{\prime}$. We refer to such a process as privacy-preserving $k$-NN (PP $k$ NN) classification over encrypted data in the cloud. Formally, we define the $\mathrm{PP} k \mathrm{NN}$ protocol as:

$$
\operatorname{PP} k \mathrm{NN}\left(D^{\prime}, q\right) \rightarrow c_{q}
$$

where $c_{q}$ denotes the class label for $q$ after applying $k$-NN classification method on $D^{\prime}$ and $q$.

\subsection{Our Contribution}

In this paper, we propose a novel PP $k \mathrm{NN}$ protocol, a secure $k$-NN classifier over semantically secure encrypted data. In our protocol, once the encrypted data are outsourced to the cloud, Alice does not participate in any computations. Therefore, no information is revealed to Alice. In particular, our protocol meets the following privacy requirements:

- Contents of $D$ or any intermediate results should not be revealed to the cloud.

- Bob's query $q$ should not be revealed to the cloud.

- $c_{q}$ should be revealed only to Bob. In addition, no information other than $c_{q}$ should be revealed to Bob.

- Data access patterns, such as the records corresponding to the $k$-nearest neighbors of $q$, should not be revealed to Bob and the cloud (to prevent any inference attacks).

We emphasize that the intermediate results seen by the cloud in our protocol are either newly generated randomized encryptions or random numbers. Thus, which data records correspond to the $k$-nearest neighbors and the output class label are not known to the cloud. In addition, after sending his encrypted query record to the cloud, Bob does not involve in any computations. Hence, data access patterns are further protected from Bob. More details are given in Section 5

The rest of the paper is organized as follows. We discuss the existing related work and some concepts as a background in Section 2, A set of privacy-preserving protocols and their possible implementations are provided in Section 3 The proposed PP $k$ NN protocol is explained in detail in Section 5 Section 6 discusses the performance of the proposed protocol based on various experiments. We conclude the paper along with future work in Section 7

\section{RELATED WORK}

In this section, we first present existing work related to privacy preserving data mining and query processing over encrypted data. Then, we present security definition and the Paillier cryptosystem along with its additive homomorphic properties. For ease of presentation, some common notations used throughout this paper are summarized in Table 1

At first, it seems fully homomorphic cryptosystems (e.g., [24]) can solve the DMED problem since it allows a third-party (that hosts the encrypted data) to execute arbitrary functions over encrypted data without ever decrypting them. However, we stress that such techniques are very expensive and their usage in practical applications have yet to 
Table 1: SOME COMMON NOTATIONS

\begin{tabular}{|l|l|}
\hline Alice & The data owner holding database $D$ \\
\hline$\left\langle E_{p k}, D_{s k}\right\rangle$ & $\begin{array}{l}\text { A pair of Paillier's encryption and decryption } \\
\text { functions with }(p k, s k) \text { as public-secret key pair }\end{array}$ \\
\hline$D^{\prime}$ & Attribute-wise encryption of $D$ \\
\hline Bob & An authorized user who can access $D^{\prime}$ in the cloud \\
\hline$q$ & Bob's input query \\
\hline$n$ & Number of data records in $D$ \\
\hline$m$ & Number of attributes in $D$ \\
\hline$w$ & Number of unique class labels in $D$ \\
\hline$l$ & $\begin{array}{l}\text { Domain size (in bits) of the Squared Euclidean } \\
\text { distance based on } D\end{array}$ \\
\hline$\left\langle z_{1}, z_{l}\right\rangle$ & The least and most significant bits of integer $z$ \\
\hline$[z]$ & Vector of encryptions of the individual bits of $z$ \\
\hline$c_{q}$ & The class label corresponding to $q$ based on $D$ \\
\hline
\end{tabular}

be explored. For example, it was shown in [25] that even for weak security parameters one "bootstrapping" operation of the homomorphic operation would take at least 30 seconds on a high performance machine.

Due to the above reason, we usually need at least two parties to perform arbitrary computations over encrypted data based on an additive homomorphic encryption scheme. It is also possible to use the existing secret sharing techniques in SMC, such as Shamir's scheme [51], to develop a PP $k N N$ protocol. However, our work is different from the secret sharing based solution from the following two aspects. (i) Solutions based on the secret sharing schemes require at least three parties whereas our work require only two parties. (ii) Hiding data access patterns is still an unsolved problem in the secret sharing based schemes, whereas our work protects data access patterns from both participating parties, and it can be extended into a solution under the secret sharing schemes. For example, the constructions based on Sharemind [8], a well-known SMC framework which is based on the secret sharing scheme, assumes that the number of participating parties is three. Thus, our work is orthogonal to Sharemind and other secret sharing based schemes. Therefore, for the rest of this paper, we omit the discussion related to the techniques that can be constructed using fully homomorphic cryptosystems or secret sharing schemes.

\subsection{Privacy-Preserving Data Mining (PPDM)}

Privacy Preserving Data Mining (PPDM) is defined as the process of extracting/deriving the knowledge about data without compromising the privacy of data [3, 41, 48]. In the past decade, many privacy-preserving classification techniques have been proposed in the literature in order to protect user privacy. Agrawal and Srikant [3], Lindell and Pinkas [40] introduced the notion of privacy-preserving under data mining applications. In particular to privacypreserving classification, the goal is to build a classifier in order to predict the class label of input data record based on the distributed training dataset without compromising the privacy of data.

1. Data Perturbation Methods: In these methods, values of individual data records are perturbed by adding random noise in a such way that the distribution of perturbed data look very different from that of actual data. After such a transformation, the perturbed data is sent to the miner to perform the desired data mining tasks. Agrawal and Srikant [3] proposed the first data perturbation technique to build a decision-tree classifier. Since then many other randomizationbased methods have been proposed in the literature such as [5, 21,22, 44,58]. However, as mentioned earlier in Section 1. data perturbation techniques cannot be applicable for semantically secure encrypted data. Also, they do not produce accurate data mining results due to the addition of statistical noises to the data.

2. Data Distribution Methods: These methods assume the dataset is partitioned either horizontally or vertically and distributed across different parties. The parties later can collaborate to securely mine the combined data and learn 
the global data mining results. During this process, data owned by individual parties is not revealed to other parties. This approach was first introduced by Lindell and Pinkas [40] who proposed a decision tree classifier under two-party setting. Since then much work has been published using secure multiparty computation techniques [1, 15, 33, 37, 55].

Classification is one important task in many applications of data mining such as health-care and business. Recently, performing data mining in the cloud attracted significant attention. In cloud computing, data owner outsources his/her data to the cloud. However, from user's perspective, privacy becomes an important issue when sensitive data needs to be outsourced to the cloud. The direct way to guard the outsourced data is to apply encryption on the data before outsourcing.

Unfortunately, since the hosted data on the cloud is in encrypted form in our problem domain, the existing privacy preserving classification techniques are not sufficient and applicable to $\mathrm{PP} k \mathrm{NN}$ due to the following reasons. $(i)$ In existing methods, the data are partitioned among at least two parties, whereas in our case encrypted data are hosted on the cloud. (ii) Since some amount of information is loss due to the addition of statistical noises in order to hide the sensitive attributes, the existing methods are not accurate. (iii) Leakage of data access patterns: the cloud can easily derive useful and sensitive information about users' data items by simply observing the database access patterns. For the same reasons, in this paper, we do not consider secure $k$-nearest neighbor techniques in which the data are distributed between two parties (e.g., [47]).

\subsection{Query processing over encrypted data}

Using encryption as a way to achieve the data confidentiality may cause another issue at the cloud during the query evaluation. The question here is "how can the cloud perform computations over encrypted data while the data stored are in encrypted form?" Along this direction, various techniques related to query processing over encrypted data have been proposed, e.g., [2, 30, 32]. However, we observe that $\mathrm{PP} k \mathrm{NN}$ is a more complex problem than the execution of simple $k \mathrm{NN}$ queries over encrypted data [53, 54]. For one, the intermediate $k$-nearest neighbors in the classification process, should not be disclosed to the cloud or any users. We emphasize that the recent method in [54] reveals the $k$-nearest neighbors to the user. Secondly, even if we know the $k$-nearest neighbors, it is still very difficult to find the majority class label among these neighbors since they are encrypted at the first place to prevent the cloud from learning sensitive information. Third, the existing work did not addressed the access pattern issue which is a crucial privacy requirement from the user's perspective.

In our most recent work [20], we proposed a novel secure $k$-nearest neighbor query protocol over encrypted data that protects data confidentiality, user's query privacy, and hides data access patterns. However, as mentioned above, $\mathrm{PP} k \mathrm{NN}$ is a more complex problem and it cannot be solved directly using the existing secure $k$-nearest neighbor techniques over encrypted data. Therefore, in this paper, we extend our previous work in [20] and provide a new solution to the $\mathrm{PP} k \mathrm{NN}$ classifier problem over encrypted data.

More specifically, this paper is different from our preliminary work [20] in the following four aspects. First, in this paper, we introduced new security primitives, namely secure minimum (SMIN), secure minimum out of $n$ numbers $\left(\mathrm{SMIN}_{n}\right)$, secure frequency $(\mathrm{SF})$, and proposed new solutions for them. Second, the work in [20] did not provide any formal security analysis of the underlying sub-protocols. On the other hand, this paper provides formal security proofs of the underlying sub-protocols as well as the PP $k \mathrm{NN}$ protocol under the semi-honest model. Additionally, we demonstrate various techniques through which the proposed protocol can possibly be extended to a protocol that is secure under the malicious model. Third, our preliminary work in [20] addresses only secure $k N N$ query which is similar to Stage 1 of PP $k$ NN. However, Stage 2 in PP $k$ NN is entirely new. Finally, our empirical analyses in Section VI are based on a real dataset whereas the results in [20] are based on a simulated dataset. In addition, new results are included in this paper.

As mentioned earlier, one can implement the proposed protocols under secret sharing schemes. By doing so, we need to have at least three independent parties. In this work, we only concentrate on the two party situation; thus, we adopted the Paillier cryptosystem. Two-party and multi-party (three or more parties) SMC protocols are complement to each other, and their applications mainly depend on the number of available participants. In practice, two mutually independent clouds are easier to find and are cheaper to operate. On the other hand, utilizing three cloud servers and secret sharing schemes to implement the proposed protocols may result more efficient running time. We believe both two-party and multi-party schemes are important. As a future work, we will consider secret sharing based $\mathrm{PP} k \mathrm{NN}$ 
implementations.

\subsection{Threat Model}

In this paper, privacy/security is closely related to the amount of information disclosed during the execution of a protocol. In the proposed protocols, our goal is to ensure no information leakage to the involved parties other than what they can deduce from their own outputs. There are many ways to define information disclosure. To maximize privacy or minimize information disclosure, we adopt the security definitions in the literature of secure multiparty computation (SMC) first introduced by Yao's Millionaires' problem for which a provably secure solution was developed [56, 57]. This was extended to multiparty computations by Goldreich et al. [28]. It was proved in [28] that any computation which can be done in polynomial time by a single party can also be done securely by multiple parties. Since then much work has been published for the multiparty case (e.g., [6, 7, 12, 13, 16, 26, 38, 39]).

There are three common adversarial models under SMC: semi-honest, covert and malicious. An adversarial model generally specifies what an adversary or attacker is allowed to do during an execution of a secure protocol. In the semi-honest model, an attacker (i.e., one of the participating parties) is expected to follow the prescribed steps of a protocol. However, the attacker can compute any additional information based on his or her private input, output and messages received during an execution of the secure protocol. As a result, whatever can be inferred from the private input and output of an attacker is not considered as a privacy violation. An adversary in the semi-honest model can be treated as a passive attacker whereas an adversary in the malicious model can be treated as an active attacker who can arbitrarily diverge from the normal execution of a protocol. On the other hand, the covert adversary model [4] lies between the semi-honest and malicious models. More specifically, an adversary under the covert model may deviate arbitrarily from the rules of a protocol, however, in the case of cheating, the honest party is guaranteed to detect this cheating with good probability.

In this paper, to develop secure and efficient protocols, we assume that parties are semi-honest for two reasons. First, as mentioned in [35], developing protocols under the semi-honest setting is an important first step towards constructing protocols with stronger security guarantees. Second, it is worth pointing out that all the practical SMC protocols proposed in the literature (e.g., [31,34,35,43]) are implemented only under the semi-honest model. By semi-honest model, we implicitly assume that the cloud service providers (or other participating users) utilized in our protocols do not collude. Since current known cloud service providers are well established IT companies, it is hard to see the possibility for two companies, e.g., Google and Amazon, to collude as it will damage their reputations and consequently place negative impact on their revenues. Thus, in our problem domain, assuming the participating parties are semi-honest is very realistic. Detailed security definitions and models can be found in [26, 27]. Briefly, the following definition captures the above discussion regarding a secure protocol under the semi-honest model.

Definition 1 Let $a_{i}$ be the input of party $P_{i}, \Pi_{i}(\pi)$ be $P_{i}$ 's execution image of the protocol $\pi$ and $b_{i}$ be the output for party $P_{i}$ computed from $\pi$. Then, $\pi$ is secure if $\Pi_{i}(\pi)$ can be simulated from $a_{i}$ and $b_{i}$ such that distribution of the simulated image is computationally indistinguishable from $\Pi_{i}(\pi)$.

In the above definition, an execution image generally includes the input, the output and the messages communicated during an execution of a protocol. To prove a protocol is secure under semi-honest model, we generally need to show that the execution image of a protocol does not leak any information regarding the private inputs of participating parties [26]. In this paper, we first propose a $\mathrm{PP} k \mathrm{NN}$ protocol that is secure under the semi-honest model. We then extend it to be secure under other adversarial models.

\subsection{Paillier Cryptosystem}

The Paillier cryptosystem is an additive homomorphic and probabilistic asymmetric encryption scheme whose security is based on the Decisional Composite Residuosity Assumption [45]. Let $E_{p k}$ be the encryption function with public key $p k$ given by $(N, g)$ and $D_{s k}$ be the decryption function with secret key $s k$ given by a trapdoor function $\lambda$ (that is, the knowledge of the factors of $N$ ). Here, $N$ is the RSA modulus of bit length $K$ and generator $g \in \mathbb{Z}_{N^{2}}^{*}$. For any given $a, b \in \mathbb{Z}_{N}$, the Paillier encryption scheme exhibits the following properties: 


\section{a. Homomorphic Addition}

$$
D_{s k}\left(E_{p k}(a+b)\right)=D_{s k}\left(E_{p k}(a) * E_{p k}(b) \bmod N^{2}\right)
$$

b. Homomorphic Multiplication

$$
D_{s k}\left(E_{p k}(a * b)\right)=D_{s k}\left(E_{p k}(a)^{b} \bmod N^{2}\right)
$$

c. Semantic Security - The encryption scheme is semantically secure [26,29]. Briefly, given a set of ciphertexts, an adversary cannot deduce any additional information regarding the corresponding plaintexts.

In this paper, we assume that a data owner encrypted his or her data using Paillier cryptosystem before outsourcing to a cloud. However, we stress that any other additive homomorphic public-key cryptosystem satisfying the above properties can also be used to implement our proposed protocol. We simply use the well-known Paillier's scheme in our implementations. Also, for ease of presentation, we drop the mod $N^{2}$ term during the homomorphic operations in the rest of this paper. In addition, many extensions to the Paillier cryptosystem have been proposed in the literature [17, 18, 23]. However, to be more specific, in this paper we use the original Paillier cryptosystem [45]. Nevertheless, our work can be directly applied to the above mentioned extensions of the Paillier's scheme.

\section{Privacy-Preserving Protocols}

In this section, we present a set of generic sub-protocols that will be used in constructing our proposed $k$-NN protocol in Section 5. All of the below protocols are considered under two-party semi-honest setting. In particular, we assume the exist of two semi-honest parties $P_{1}$ and $P_{2}$ such that the Paillier's secret key $s k$ is known only to $P_{2}$ whereas $p k$ is treated as public.

- Secure Multiplication (SM) Protocol:

This protocol considers $P_{1}$ with input $\left(E_{p k}(a), E_{p k}(b)\right)$ and outputs $E_{p k}(a * b)$ to $P_{1}$, where $a$ and $b$ are not known to $P_{1}$ and $P_{2}$. During this process, no information regarding $a$ and $b$ is revealed to $P_{1}$ and $P_{2}$.

- Secure Squared Euclidean Distance (SSED) Protocol:

In this protocol, $P_{1}$ with input $\left(E_{p k}(X), E_{p k}(Y)\right)$ and $P_{2}$ with $s k$ securely compute the encryption of squared Euclidean distance between vectors $X$ and $Y$. Here $X$ and $Y$ are $m$ dimensional vectors where $E_{p k}(X)=$ $\left\langle E_{p k}\left(x_{1}\right), \ldots, E_{p k}\left(x_{m}\right)\right\rangle$ and $E_{p k}(Y)=\left\langle E_{p k}\left(y_{1}\right), \ldots, E_{p k}\left(y_{m}\right)\right\rangle$. The output of the SSED protocol is $E_{p k}(\mid X-$ $\left.Y\right|^{2}$ ) which is known only to $P_{1}$.

- Secure Bit-Decomposition (SBD) Protocol:

$P_{1}$ with input $E_{p k}(z)$ and $P_{2}$ securely compute the encryptions of the individual bits of $z$, where $0 \leq z<2^{l}$. The output $[z]=\left\langle E_{p k}\left(z_{1}\right), \ldots, E_{p k}\left(z_{l}\right)\right\rangle$ is known only to $P_{1}$. Here $z_{1}$ and $z_{l}$ are the most and least significant bits of integer $z$, respectively.

- Secure Minimum (SMIN) Protocol:

In this protocol, $P_{1}$ holds private input $\left(u^{\prime}, v^{\prime}\right)$ and $P_{2}$ holds $s k$, where $u^{\prime}=\left([u], E_{p k}\left(s_{u}\right)\right)$ and $v^{\prime}=\left([v], E_{p k}\left(s_{v}\right)\right)$. Here $s_{u}$ (resp., $s_{v}$ ) denotes the secret associated with $u$ (resp., $v$ ). The goal of SMIN is for $P_{1}$ and $P_{2}$ to jointly compute the encryptions of the individual bits of minimum number between $u$ and $v$. In addition, they compute $E_{p k}\left(s_{\min (u, v)}\right)$. That is, the output is $\left([\min (u, v)], E_{p k}\left(s_{\min (u, v)}\right)\right)$ which will be known only to $P_{1}$. During this protocol, no information regarding the contents of $u, v, s_{u}$, and $s_{v}$ is revealed to $P_{1}$ and $P_{2}$.

- Secure Minimum out of $n$ Numbers $\left(\operatorname{SMIN}_{n}\right)$ Protocol:

In this protocol, we consider $P_{1}$ with $n$ encrypted vectors $\left(\left[d_{1}\right], \ldots,\left[d_{n}\right]\right)$ along with their respective encrypted secrets and $P_{2}$ with $s k$. Here $\left[d_{i}\right]=\left\langle E_{p k}\left(d_{i, 1}\right), \ldots, E_{p k}\left(d_{i, l}\right)\right\rangle$ where $d_{i, 1}$ and $d_{i, l}$ are the most and least significant bits of integer $d_{i}$ respectively, for $1 \leq i \leq n$. The secret of $d_{i}$ is given by $s_{d_{i}} . P_{1}$ and $P_{2}$ jointly compute $\left[\min \left(d_{1}, \ldots, d_{n}\right)\right]$. In addition, they compute $E_{p k}\left(s_{\min \left(d_{1}, \ldots, d_{n}\right)}\right)$. At the end of this protocol, the output $\left(\left[\min \left(d_{1}, \ldots, d_{n}\right)\right], E_{p k}\left(s_{\min \left(d_{1}, \ldots, d_{n}\right)}\right)\right)$ is known only to $P_{1}$. During the $\mathrm{SMIN}_{n}$ protocol, no information regarding any of $d_{i}$ 's and their secrets is revealed to $P_{1}$ and $P_{2}$. 


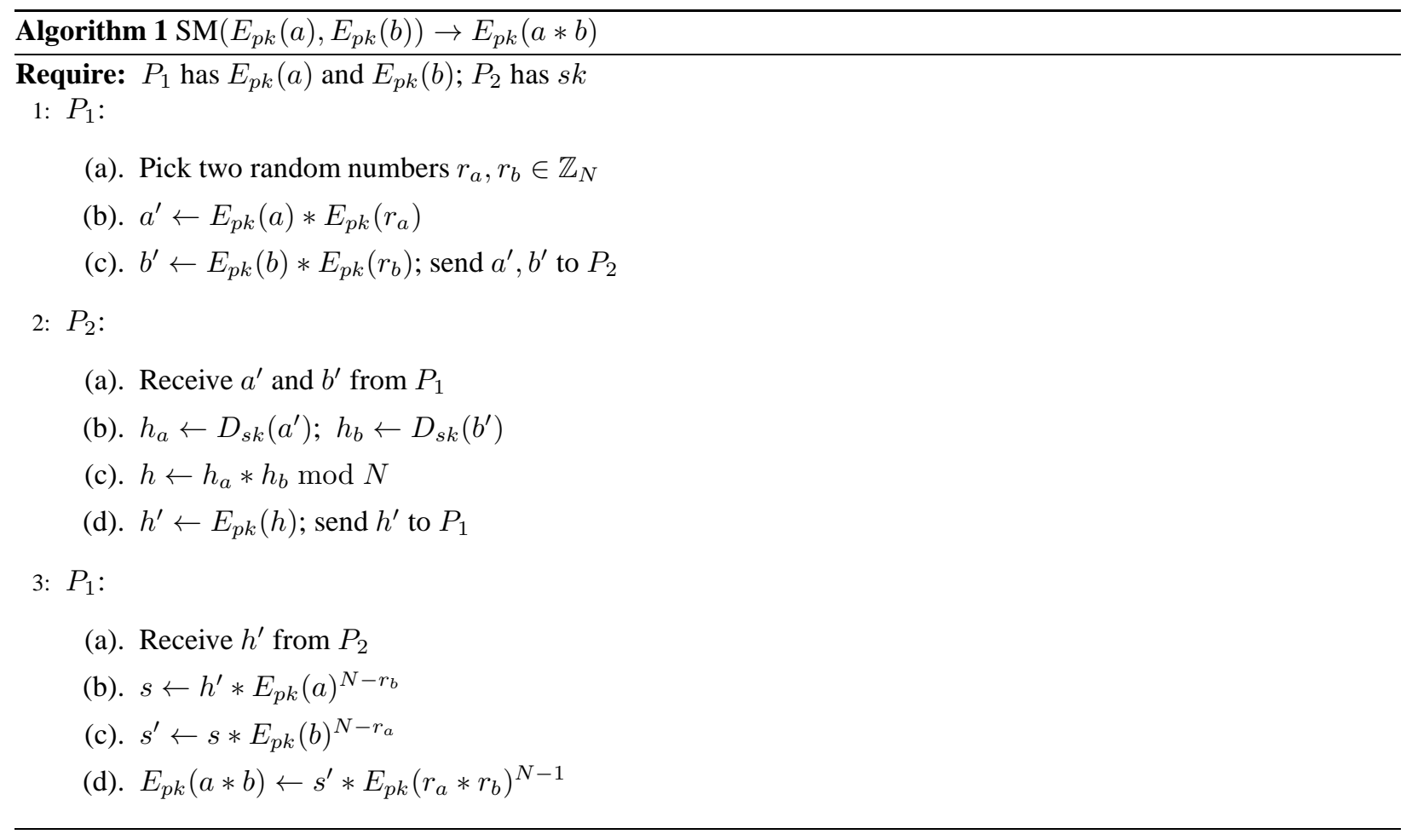

- Secure Bit-OR (SBOR) Protocol:

$P_{1}$ with input $\left(E_{p k}\left(o_{1}\right), E_{p k}\left(o_{2}\right)\right)$ and $P_{2}$ securely compute $E_{p k}\left(o_{1} \vee o_{2}\right)$, where $o_{1}$ and $o_{2}$ are two bits. The output $E_{p k}\left(o_{1} \vee o_{2}\right)$ is known only to $P_{1}$.

- Secure Frequency (SF) Protocol:

In this protocol, $P_{1}$ with private input $\left(\left\langle E_{p k}\left(c_{1}\right), \ldots E_{p k}\left(c_{w}\right)\right\rangle,\left\langle E_{p k}\left(c_{1}^{\prime}\right), \ldots, E_{p k}\left(c_{k}^{\prime}\right)\right\rangle\right)$ and $P_{2}$ securely compute the encryption of the frequency of $c_{j}$, denoted by $f\left(c_{j}\right)$, in the list $\left\langle c_{1}^{\prime}, \ldots, c_{k}^{\prime}\right\rangle$, for $1 \leq j \leq w$. We explicitly assume that $c_{j}$ 's are unique and $c_{i}^{\prime} \in\left\{c_{1}, \ldots, c_{w}\right\}$, for $1 \leq i \leq k$. The output $\left\langle E_{p k}\left(f\left(c_{1}\right)\right), \ldots, E_{p k}\left(f\left(c_{w}\right)\right)\right\rangle$ will be known only to $P_{1}$. During the SF protocol, no information regarding $c_{i}^{\prime}, c_{j}$, and $f\left(c_{j}\right)$ is revealed to $P_{1}$ and $P_{2}$, for $1 \leq i \leq k$ and $1 \leq j \leq w$.

Now we either propose a new solution or refer to the most efficient known implementation to each of the above protocols. First of all, efficient solutions to SM, SSED, SBD and SBOR were presented in our preliminary work [20]. However, for completeness, we briefly discuss those solutions here. Also, we discuss SMIN, $\mathrm{SMIN}_{n}$, and SF problems in detail and propose new solutions to each one of them.

Secure Multiplication (SM). Consider a party $P_{1}$ with private input $\left(E_{p k}(a), E_{p k}(b)\right)$ and a party $P_{2}$ with the secret key $s k$. The goal of the secure multiplication (SM) protocol is to return the encryption of $a * b$, i.e., $E_{p k}(a * b)$ as output to $P_{1}$. During this protocol, no information regarding $a$ and $b$ is revealed to $P_{1}$ and $P_{2}$. The basic idea of the $\mathrm{SM}$ protocol is based on the following property which holds for any given $a, b \in \mathbb{Z}_{N}$ :

$$
a * b=\left(a+r_{a}\right) *\left(b+r_{b}\right)-a * r_{b}-b * r_{a}-r_{a} * r_{b}
$$

where all the arithmetic operations are performed under $\mathbb{Z}_{N}$. The overall steps in SM are shown in Algorithm 1 . Briefly, $P_{1}$ initially randomizes $a$ and $b$ by computing $a^{\prime}=E_{p k}(a) * E_{p k}\left(r_{a}\right)$ and $b^{\prime}=E_{p k}(b) * E_{p k}\left(r_{b}\right)$, and sends them to $P_{2}$. Here $r_{a}$ and $r_{b}$ are random numbers in $\mathbb{Z}_{N}$ known only to $P_{1}$. Upon receiving, $P_{2}$ decrypts and multiplies them to get $h=\left(a+r_{a}\right) *\left(b+r_{b}\right) \bmod N$. Then, $P_{2}$ encrypts $h$ and sends it to $P_{1}$. After this, $P_{1}$ removes extra random factors from $h^{\prime}=E_{p k}\left(\left(a+r_{a}\right) *\left(b+r_{b}\right)\right)$ based on Equation 1 to get $E_{p k}(a * b)$. Note that, under Paillier 


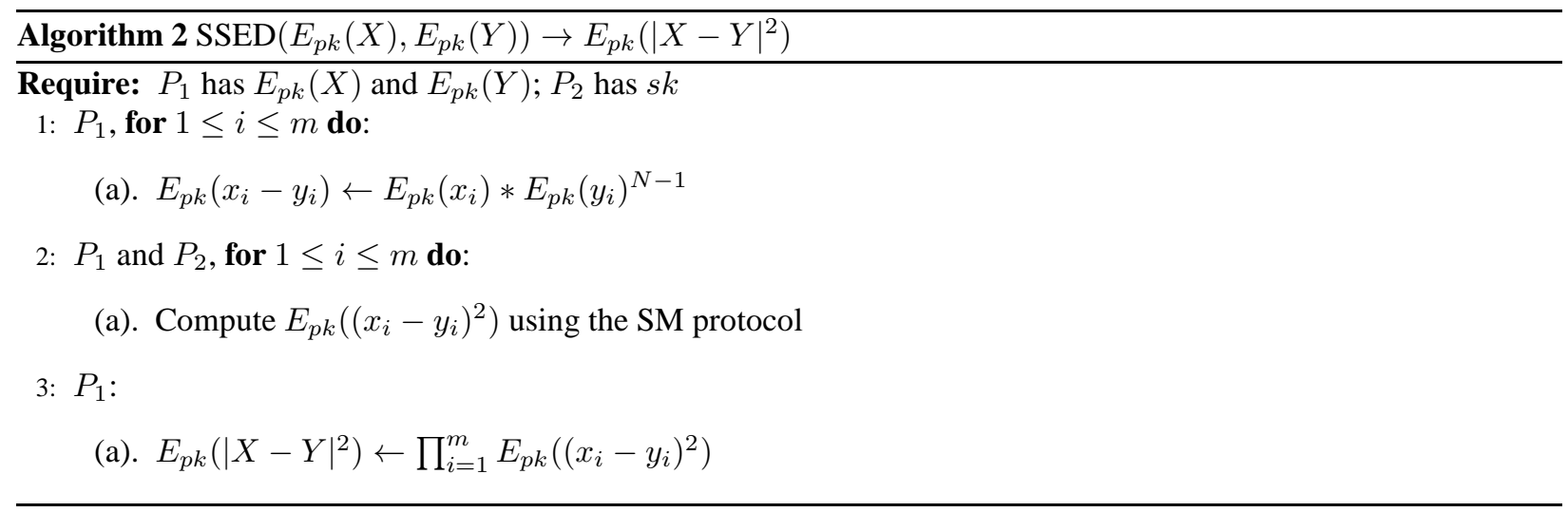

cryptosystem, " $N-x$ " is equivalent to " $-x$ " in $\mathbb{Z}_{N}$. Hereafter, we use the notation $r \in_{R} \mathbb{Z}_{N}$ to denote $r$ as a random number in $\mathbb{Z}_{N}$.

Example 2 Let us assume that $a=59$ and $b=58$. For simplicity, let $r_{a}=1$ and $r_{b}=3$. Initially, $P_{1}$ computes $a^{\prime}=E_{p k}(60)=E_{p k}(a) * E_{p k}\left(r_{a}\right), b^{\prime}=E_{p k}(61)=E_{p k}(b) * E_{p k}\left(r_{b}\right)$ and sends them to $P_{2}$. Then, $P_{2}$ decrypts and multiplies them to get $h=3660$. After this, $P_{2}$ encrypts $h$ to get $h^{\prime}=E_{p k}(3660)$ and sends it to $P_{1}$. Upon receiving $h^{\prime}, P_{1}$ computes $s=E_{p k}(3483)=E_{p k}\left(3660-a * r_{b}\right)$, and $s^{\prime}=E_{p k}(3425)=E_{p k}\left(3483-b * r_{a}\right)$. Finally, $P_{1}$ computes $E_{p k}(a * b)=E_{p k}(3422)=E_{p k}\left(3425-r_{a} * r_{b}\right)$.

Secure Squared Euclidean Distance (SSED). In the SSED protocol, $P_{1}$ holds two encrypted vectors $\left(E_{p k}(X), E_{p k}(Y)\right)$ and $P_{2}$ holds the secret key $s k$. Here $X$ and $Y$ are two $m$-dimensional vectors where $E_{p k}(X)=\left\langle E_{p k}\left(x_{1}\right), \ldots, E_{p k}\left(x_{m}\right)\right\rangle$ and $E_{p k}(Y)=\left\langle E_{p k}\left(y_{1}\right), \ldots, E_{p k}\left(y_{m}\right)\right\rangle$. The goal of the SSED protocol is to securely compute $E_{p k}\left(|X-Y|^{2}\right)$, where $|X-Y|$ denotes the Euclidean distance between vectors $X$ and $Y$. At a high level, the basic idea of SSED follows from following equation:

$$
|X-Y|^{2}=\sum_{i=1}^{m}\left(x_{i}-y_{i}\right)^{2}
$$

The main steps involved in the SSED protocol are as shown in Algorithm 2 Briefly, for $1 \leq i \leq m, P_{1}$ initially computes $E_{p k}\left(x_{i}-y_{i}\right)$ by using the homomorphic properties. Then $P_{1}$ and $P_{2}$ jointly compute $E_{p k}\left(\left(x_{i}-y_{i}\right)^{2}\right)$ using the SM protocol, for $1 \leq i \leq m$. Note that the outputs of SM are known only to $P_{1}$. Finally, by applying homomorphic properties on $E_{p k}\left(\left(x_{i}-y_{i}\right)^{2}\right), P_{1}$ computes $E_{p k}\left(|X-Y|^{2}\right)$ locally based on Equation 2

Example 3 Let us assume that $P_{1}$ holds the encrypted data records of $X$ and $Y$ given by $E_{p k}(X)=\left\langle E_{p k}(63), E_{p k}(1)\right.$, $\left.E_{p k}(1), E_{p k}(145), E_{p k}(233), E_{p k}(1), E_{p k}(3), E_{p k}(0), E_{p k}(6), E_{p k}(0)\right\rangle$ and $E_{p k}(Y)=\left\langle E_{p k}(56), E_{p k}(1), E_{p k}(3)\right.$, $\left.E_{p k}(130), E_{p k}(256), E_{p k}(1), E_{p k}(2), E_{p k}(1), E_{p k}(6), E_{p k}(2)\right\rangle$. During the SSED protocol, $P_{1}$ initially computes $E_{p k}\left(x_{1}-y_{1}\right)=E_{p k}(7), \ldots, E_{p k}\left(x_{10}-y_{10}\right)=E_{p k}(-2)$. Then, $P_{1}$ and $P_{2}$ jointly compute $E_{p k}\left(\left(x_{1}-y_{1}\right)^{2}\right)=$ $E_{p k}(49)=S M\left(E_{p k}(7), E_{p k}(7)\right), \ldots, E_{p k}\left(\left(x_{10}-y_{10}\right)^{2}\right)=S M\left(E_{p k}(-2), E_{p k}(-2)\right)=E_{p k}(4) . P_{1}$ locally computes $E_{p k}\left(|X-Y|^{2}\right)=E_{p k}\left(\sum_{i=1}^{10}\left(x_{i}-y_{i}\right)^{2}\right)=E_{p k}(813)$.

Secure Bit-Decomposition (SBD). We assume that $P_{1}$ has $E_{p k}(z)$ and $P_{2}$ has $s k$, where $z$ is not known to both parties and $0 \leq z<2^{l}$. Given $E_{p k}(z)$, the goal of the secure bit-decomposition (SBD) protocol is to compute the encryptions of the individual bits of binary representation of $z$. That is, the output is $[z]=\left\langle E_{p k}\left(z_{1}\right), \ldots, E_{p k}\left(z_{l}\right)\right\rangle$, where $z_{1}$ and $z_{l}$ denote the most and least significant bits of $z$ respectively. At the end, the output $[z]$ is known only to $P_{1}$. During this process, neither the value of $z$ nor any $z_{i}$ 's is revealed to $P_{1}$ and $P_{2}$.

Since the goal of this paper is not to investigate existing SBD protocols, we simply use the most efficient SBD protocol that was recently proposed in [50]. 
Example 4 Let us assume that $z=55$ and $l=6$. Then the $S B D$ protocol in [50] with private input $E_{p k}(55)$ returns $[55]=\left\langle E_{p k}(1), E_{p k}(1), E_{p k}(0), E_{p k}(1), E_{p k}(1), E_{p k}(1)\right\rangle$ as the output to $P_{1}$.

Secure Minimum (SMIN). In this protocol, we assume that $P_{1}$ holds private input $\left(u^{\prime}, v^{\prime}\right)$ and $P_{2}$ holds $s k$, where $u^{\prime}=\left([u], E_{p k}\left(s_{u}\right)\right)$ and $v^{\prime}=\left([v], E_{p k}\left(s_{v}\right)\right)$. Here $s_{u}$ and $s_{v}$ denote the secrets corresponding to $u$ and $v$, respectively. The main goal of SMIN is to securely compute the encryptions of the individual bits of $\min (u, v)$, denoted by $[\min (u, v)]$. Here $[u]=\left\langle E_{p k}\left(u_{1}\right), \ldots, E_{p k}\left(u_{l}\right)\right\rangle$ and $[v]=\left\langle E_{p k}\left(v_{1}\right), \ldots, E_{p k}\left(v_{l}\right)\right\rangle$, where $u_{1}$ (resp., $\left.v_{1}\right)$ and $u_{l}$ (resp., $v_{l}$ ) are the most and least significant bits of $u$ (resp., $v$ ), respectively. In addition, they compute $E_{p k}\left(s_{\min (u, v)}\right)$, the encryption of the secret corresponding to the minimum value between $u$ and $v$. At the end of SMIN, the output $\left([\min (u, v)], E_{p k}\left(s_{\min (u, v)}\right)\right)$ is known only to $P_{1}$.

We assume that $0 \leq u, v<2^{l}$ and propose a novel SMIN protocol. Our solution to SMIN is mainly motivated from the work of [20]. Precisely, the basic idea of the proposed SMIN protocol is for $P_{1}$ to randomly choose the functionality $F$ (by flipping a coin), where $F$ is either $u>v$ or $v>u$, and to obliviously execute $F$ with $P_{2}$. Since $F$ is randomly chosen and known only to $P_{1}$, the result of the functionality $F$ is oblivious to $P_{2}$. Based on the comparison result and chosen $F, P_{1}$ computes $[\min (u, v)]$ and $E_{p k}\left(s_{\min (u, v)}\right)$ locally using homomorphic properties.

The overall steps involved in the SMIN protocol are shown in Algorithm 3 . To start with, $P_{1}$ initially chooses the functionality $F$ as either $u>v$ or $v>u$ randomly. Then, using the SM protocol, $P_{1}$ computes $E_{p k}\left(u_{i} * v_{i}\right)$ with the help of $P_{2}$, for $1 \leq i \leq l$. After this, the protocol has the following key steps, performed by $P_{1}$ locally, for $1 \leq i \leq l$ :

- Compute the encrypted bit-wise XOR between the bits $u_{i}$ and $v_{i}$ as $T_{i}=E_{p k}\left(u_{i} \oplus v_{i}\right)$ using the below formulation]:

$$
T_{i}=E_{p k}\left(u_{i}\right) * E_{p k}\left(v_{i}\right) * E_{p k}\left(u_{i} * v_{i}\right)^{N-2}
$$

- Compute an encrypted vector $H$ by preserving the first occurrence of $E_{p k}(1)$ (if there exists one) in $T$ by initializing $H_{0}=E_{p k}(0)$. The rest of the entries of $H$ are computed as $H_{i}=H_{i-1}^{r_{i}} * T_{i}$. We emphasize that at most one of the entry in $H$ is $E_{p k}(1)$ and the remaining entries are encryptions of either 0 or a random number.

- Then, $P_{1}$ computes $\Phi_{i}=E_{p k}(-1) * H_{i}$. Note that "- 1 " is equivalent to " $N-1$ " under $\mathbb{Z}_{N}$. From the above discussions, it is clear that $\Phi_{i}=E_{p k}(0)$ at most once since $H_{i}$ is equal to $E_{p k}(1)$ at most once. Also, if $\Phi_{j}=E_{p k}(0)$, then index $j$ is the position at which the bits of $u$ and $v$ differ first (starting from the most significant bit position).

Now, depending on $F, P_{1}$ creates two encrypted vectors $W$ and $\Gamma$ as follows, for $1 \leq i \leq l$ :

- If $F: u>v$, compute

$$
\begin{aligned}
W_{i} & =E_{p k}\left(u_{i}\right) * E_{p k}\left(u_{i} * v_{i}\right)^{N-1} \\
& =E_{p k}\left(u_{i} *\left(1-v_{i}\right)\right) \\
\Gamma_{i} & =E_{p k}\left(v_{i}-u_{i}\right) * E_{p k}\left(\hat{r}_{i}\right) \\
& =E_{p k}\left(v_{i}-u_{i}+\hat{r}_{i}\right)
\end{aligned}
$$

- If $F: v>u$, compute:

$$
\begin{aligned}
W_{i} & =E_{p k}\left(v_{i}\right) * E_{p k}\left(u_{i} * v_{i}\right)^{N-1} \\
& =E_{p k}\left(v_{i} *\left(1-u_{i}\right)\right) \\
\Gamma_{i} & =E_{p k}\left(u_{i}-v_{i}\right) * E_{p k}\left(\hat{r}_{i}\right) \\
& =E_{p k}\left(u_{i}-v_{i}+\hat{r}_{i}\right)
\end{aligned}
$$

\footnotetext{
${ }^{1}$ In general, for any two given bits $o_{1}$ and $o_{2}$, the property $o_{1} \oplus o_{2}=o_{1}+o_{2}-2\left(o_{1} * o_{2}\right)$ always hold.
} 


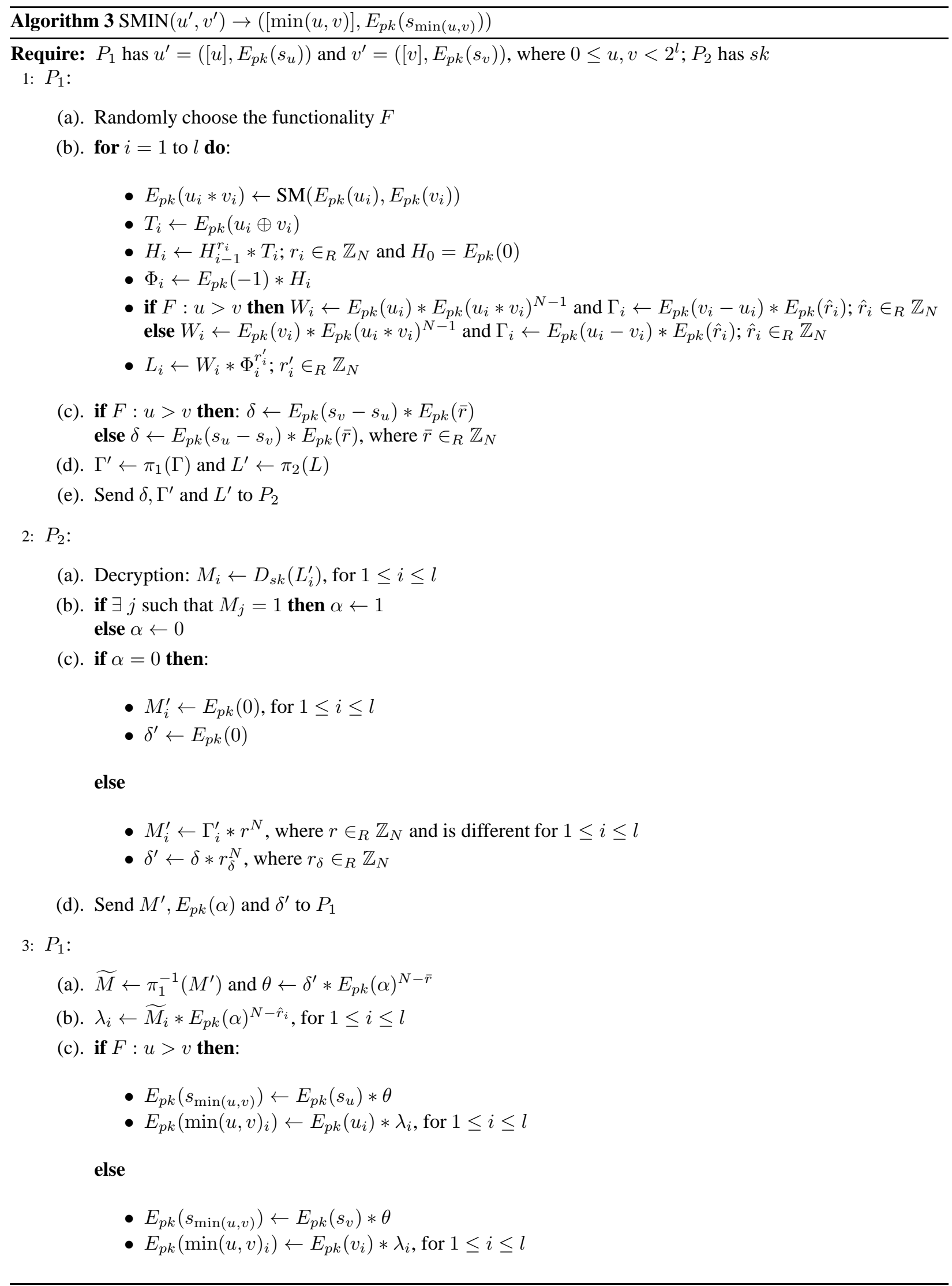


Table 2: $P_{1}$ chooses $F$ as $v>u$ where $u=55$ and $v=58$ (Note: All column values are in encrypted form except $M_{i}$ column. Also, $r \in_{R} \mathbb{Z}_{N}$ is different for each row and column. )

\begin{tabular}{ccccccccccccc}
\hline$[u]$ & {$[v]$} & $W_{i}$ & $\Gamma_{i}$ & $G_{i}$ & $H_{i}$ & $\Phi_{i}$ & $L_{i}$ & $\Gamma_{i}{ }^{\prime}$ & $L_{i}^{\prime}$ & $M_{i}$ & $\lambda_{i}$ & $\min _{i}$ \\
\hline 1 & 1 & 0 & $r$ & 0 & 0 & -1 & $r$ & $1+r$ & $r$ & $r$ & 0 & 1 \\
1 & 1 & 0 & $r$ & 0 & 0 & -1 & $r$ & $r$ & $r$ & $r$ & 0 & 1 \\
0 & 1 & 1 & $-1+r$ & 1 & 1 & 0 & 1 & $1+r$ & $r$ & $r$ & -1 & 0 \\
1 & 0 & 0 & $1+r$ & 1 & $r$ & $r$ & $r$ & $-1+r$ & $r$ & $r$ & 1 & 1 \\
1 & 1 & 0 & $r$ & 0 & $r$ & $r$ & $r$ & $r$ & 1 & 1 & 0 & 1 \\
1 & 0 & 0 & $1+r$ & 1 & $r$ & $r$ & $r$ & $r$ & $r$ & $r$ & 1 & 1 \\
\hline
\end{tabular}

where $\hat{r}_{i}$ is a random number in $\mathbb{Z}_{N}$. The observation here is if $F: u>v$, then $W_{i}=E_{p k}(1)$ iff $u_{i}>v_{i}$, and $W_{i}=E_{p k}(0)$ otherwise. Similarly, when $F: v>u$, we have $W_{i}=E_{p k}(1)$ iff $v_{i}>u_{i}$, and $W_{i}=E_{p k}(0)$ otherwise. Also, depending of $F, \Gamma_{i}$ stores the encryption of randomized difference between $u_{i}$ and $v_{i}$ which will be used in later computations.

After this, $P_{1}$ computes $L$ by combining $\Phi$ and $W$. More precisely, $P_{1}$ computes $L_{i}=W_{i} * \Phi_{i}^{r_{i}^{\prime}}$, where $r_{i}^{\prime}$ is a random number in $\mathbb{Z}_{N}$. The observation here is if $\exists$ an index $j$ such that $\Phi_{j}=E_{p k}(0)$, denoting the first flip in the bits of $u$ and $v$, then $W_{j}$ stores the corresponding desired information, i.e., whether $u_{j}>v_{j}$ or $v_{j}>u_{j}$ in encrypted form. In addition, depending on $F, P_{1}$ computes the encryption of randomized difference between $s_{u}$ and $s_{v}$ and stores it in $\delta$. Specifically, if $F: u>v$, then $\delta=E_{p k}\left(s_{v}-s_{u}+\bar{r}\right)$. Otherwise, $\delta=E_{p k}\left(s_{u}-s_{v}+\bar{r}\right)$, where $\bar{r} \in_{R} \mathbb{Z}_{N}$.

After this, $P_{1}$ permutes the encrypted vectors $\Gamma$ and $L$ using two random permutation functions $\pi_{1}$ and $\pi_{2}$. Specifically, $P_{1}$ computes $\Gamma^{\prime}=\pi_{1}(\Gamma)$ and $L^{\prime}=\pi_{2}(L)$, and sends them along with $\delta$ to $P_{2}$. Upon receiving, $P_{2}$ decrypts $L^{\prime}$ component-wise to get $M_{i}=D_{s k}\left(L_{i}^{\prime}\right)$, for $1 \leq i \leq l$, and checks for index $j$. That is, if $M_{j}=1$, then $P_{2}$ sets $\alpha$ to 1 , otherwise sets it to 0 . In addition, $P_{2}$ computes a new encrypted vector $M^{\prime}$ depending on the value of $\alpha$. Precisely, if $\alpha=0$, then $M_{i}^{\prime}=E_{p k}(0)$, for $1 \leq i \leq l$. Here $E_{p k}(0)$ is different for each $i$. On the other hand, when $\alpha=1, P_{2}$ sets $M_{i}^{\prime}$ to the re-randomized value of $\Gamma_{i}^{\prime}$. That is, $M_{i}^{\prime}=\Gamma_{i}^{\prime} * r^{N}$, where the term $r^{N}$ comes from re-randomization and $r \in_{R} \mathbb{Z}_{N}$ should be different for each $i$. Furthermore, $P_{2}$ computes $\delta^{\prime}=E_{p k}(0)$ if $\alpha=0$. However, when $\alpha=1$, $P_{2}$ sets $\delta^{\prime}$ to $\delta * r_{\delta}^{N}$, where $r_{\delta}$ is a random number in $\mathbb{Z}_{N}$. Then, $P_{2}$ sends $M^{\prime}, E_{p k}(\alpha)$ and $\delta^{\prime}$ to $P_{1}$. After receiving $M^{\prime}, E_{p k}(\alpha)$ and $\delta^{\prime}, P_{1}$ computes the inverse permutation of $M^{\prime}$ as $\widetilde{M}=\pi_{1}^{-1}\left(M^{\prime}\right)$. Then, $P_{1}$ performs the following homomorphic operations to compute the encryption of $i^{t h}$ bit of $\min (u, v)$, i.e., $E_{p k}\left(\min (u, v)_{i}\right)$, for $1 \leq i \leq l$ :

- Remove the randomness from $\widetilde{M}_{i}$ by computing $\lambda_{i}=\widetilde{M}_{i} * E_{p k}(\alpha)^{N-\hat{r}_{i}}$

- If $F: u>v$, compute the $i^{t h}$ encrypted bit of $\min (u, v)$ as $E_{p k}\left(\min (u, v)_{i}\right)=E_{p k}\left(u_{i}\right) * \lambda_{i}=E_{p k}\left(u_{i}+\alpha *\right.$ $\left.\left(v_{i}-u_{i}\right)\right)$. Otherwise, compute $E_{p k}\left(\min (u, v)_{i}\right)=E_{p k}\left(v_{i}\right) * \lambda_{i}=E_{p k}\left(v_{i}+\alpha *\left(u_{i}-v_{i}\right)\right)$.

Also, depending on $F, P_{1}$ computes $E_{p k}\left(s_{\min (u, v)}\right)$ as follows. If $F: u>v, P_{1}$ computes $E_{p k}\left(s_{\min (u, v)}\right)=$ $E_{p k}\left(s_{u}\right) * \theta$, where $\theta=\delta^{\prime} * E_{p k}(\alpha)^{N-\bar{r}}$. Otherwise, he/she computes $E_{p k}\left(s_{\min (u, v)}\right)=E_{p k}\left(s_{v}\right) * \theta$.

In the SMIN protocol, one main observation (upon which we can also justify the correctness of the final output) is that if $F: u>v$, then $\min (u, v)_{i}=(1-\alpha) * u_{i}+\alpha * v_{i}$ always holds, for $1 \leq i \leq l$. On the other hand, if $F: v>u$, then $\min (u, v)_{i}=\alpha * u_{i}+(1-\alpha) * v_{i}$ always holds. Similar conclusions can be drawn for $s_{\min (u, v) \text {. }}$ We emphasize that using similar formulations one can also design a SMAX protocol to compute $[\max (u, v)]$ and $E_{p k}\left(s_{\max (u, v)}\right)$. Also, we stress that there can be multiple secrets of $u$ and $v$ that can be fed as input (in encrypted form) to SMIN and SMAX. For example, let $s_{u}^{1}$ and $s_{u}^{2}$ (resp., $s_{v}^{1}$ and $s_{v}^{2}$ ) be two secrets associated with $u$ (resp., $v$ ). Then the SMIN protocol takes $\left([u], E_{p k}\left(s_{u}^{1}\right), E_{p k}\left(s_{u}^{2}\right)\right)$ and $\left([v], E_{p k}\left(s_{v}^{1}\right), E_{p k}\left(s_{v}^{2}\right)\right)$ as $P_{1}$ 's private input and outputs $[\min (u, v)], E_{p k}\left(s_{\min (u, v)}^{1}\right)$ and $E_{p k}\left(s_{\min (u, v)}^{2}\right)$ to $P_{1}$.

Example 5 For simplicity, consider that $u=55, v=58$, and $l=6$. Suppose $s_{u}$ and $s_{v}$ be the secrets associated 


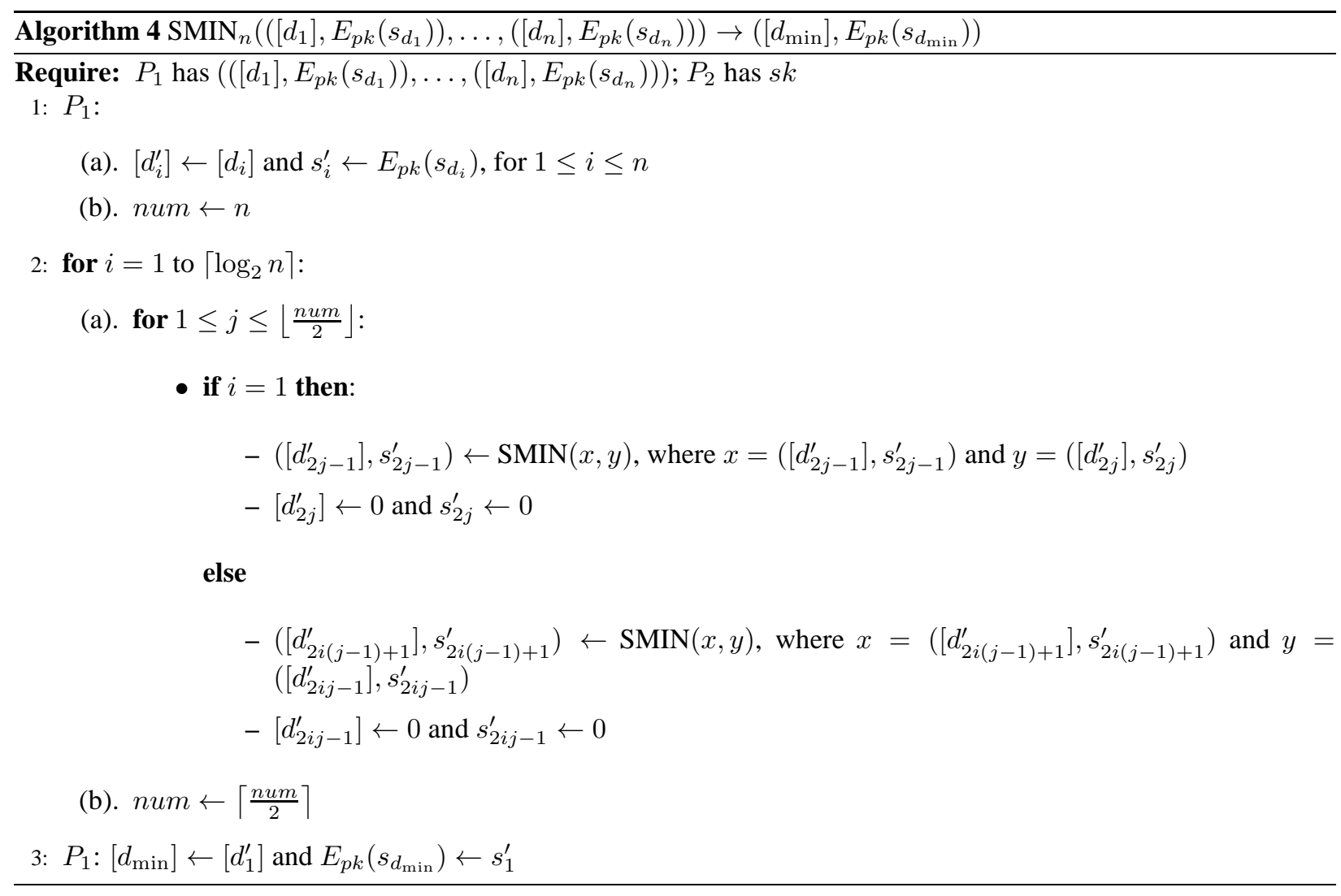

with $u$ and $v$, respectively. Assume that $P_{1}$ holds $\left([55], E_{p k}\left(s_{u}\right)\right)\left([58], E_{p k}\left(s_{v}\right)\right)$. In addition, we assume that $P_{1}$ 's random permutation functions are as given below.

$\begin{array}{llllllll}i & = & 1 & 2 & 3 & 4 & 5 & 6 \\ \pi_{1}(i) & = & \downarrow & \downarrow & \downarrow & \downarrow & \downarrow & \downarrow \\ \pi_{2}(i) & = & 2 & 1 & 5 & 6 & 3 & 4\end{array}$

Without loss of generality, suppose $P_{1}$ chooses the functionality $F: v>u$. Then, various intermediate results based on the SMIN protocol are as shown in Table 2 Following from Table 2 we observe that:

- At most one of the entry in $H$ is $E_{p k}(1)$, namely $H_{3}$, and the remaining entries are encryptions of either 0 or a random number in $\mathbb{Z}_{N}$.

- Index $j=3$ is the first position at which the corresponding bits of $u$ and $v$ differ.

- $\Phi_{3}=E_{p k}(0)$ since $H_{3}$ is equal to $E_{p k}(1)$. Also, since $M_{5}=1, P_{2}$ sets $\alpha$ to 1 .

- In addition, $E_{p k}\left(s_{\min (u, v)}\right)=E_{p k}\left(\alpha * s_{u}+(1-\alpha) * s_{v}\right)=E_{p k}\left(s_{u}\right)$.

At the end of SMIN, only $P_{1}$ knows $[\min (u, v)]=[u]=[55]$ and $E_{p k}\left(s_{\min (u, v)}\right)=E_{p k}\left(s_{u}\right)$.

Secure Minimum out of $n$ Numbers $\left(\mathbf{S M I N}_{n}\right)$. Consider $P_{1}$ with private input $\left(\left[d_{1}\right], \ldots,\left[d_{n}\right]\right)$ along with their encrypted secrets and $P_{2}$ with $s k$, where $0 \leq d_{i}<2^{l}$ and $\left[d_{i}\right]=\left\langle E_{p k}\left(d_{i, 1}\right), \ldots, E_{p k}\left(d_{i, l}\right)\right\rangle$, for $1 \leq i \leq n$. 


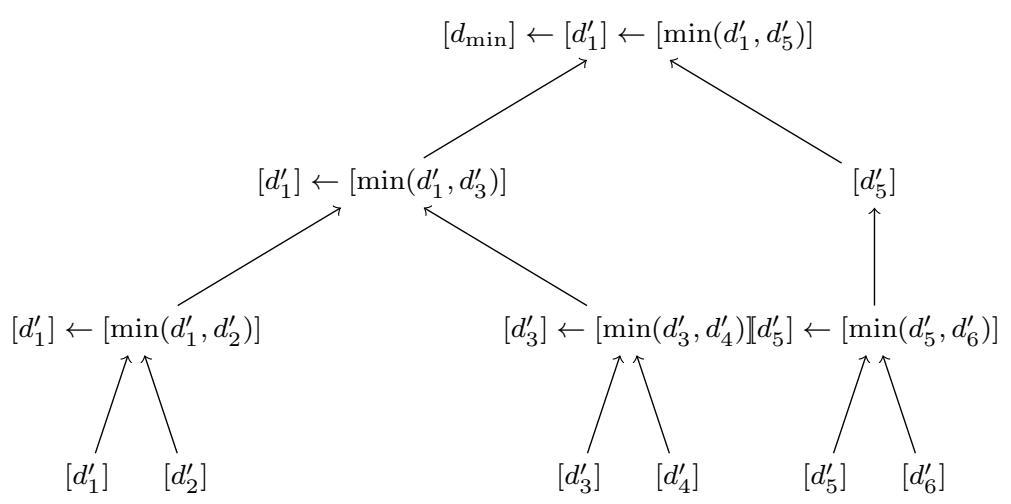

Figure 1: Binary execution tree for $n=6$ based on $\operatorname{SMIN}_{n}$

Here the secret of $d_{i}$ is denoted by $E_{p k}\left(s_{d_{i}}\right)$, for $1 \leq i \leq n$. The main goal of the $\mathrm{SMIN}_{n}$ protocol is to compute $\left[\min \left(d_{1}, \ldots, d_{n}\right)\right]=\left[d_{\min }\right]$ without revealing any information about $d_{i}$ 's to $P_{1}$ and $P_{2}$. In addition, they compute the encryption of the secret corresponding to the global minimum, denoted by $E_{p k}\left(s_{d_{\min }}\right)$. Here we construct a new $\mathrm{SMIN}_{n}$ protocol by utilizing SMIN as the building block. The proposed SMIN $_{n}$ protocol is an iterative approach and it computes the desired output in an hierarchical fashion. In each iteration, minimum between a pair of values and the secret corresponding to the minimum value are computed (in encrypted form) and fed as input to the next iteration, thus, generating a binary execution tree in a bottom-up fashion. At the end, only $P_{1}$ knows the final result $\left[d_{\min }\right]$ and $E_{p k}\left(s_{d_{\min }}\right)$.

The overall steps involved in the proposed $\mathrm{SMIN}_{n}$ protocol are highlighted in Algorithm 4 Initially, $P_{1}$ assigns $\left[d_{i}\right]$ and $E_{p k}\left(s_{d_{i}}\right)$ to a temporary vector $\left[d_{i}^{\prime}\right]$ and variable $s_{i}^{\prime}$, for $1 \leq i \leq n$, respectively. Also, he/she creates a global variable num and initializes it to $n$, where num represents the number of (non-zero) vectors involved in each iteration. Since the $\mathrm{SMIN}_{n}$ protocol executes in a binary tree hierarchy (bottom-up fashion), we have $\left\lceil\log _{2} n\right\rceil$ iterations, and in each iteration, the number of vectors involved varies. In the first iteration (i.e., $i=1), P_{1}$ with private input $\left(\left(\left[d_{2 j-1}^{\prime}\right], s_{2 j-1}^{\prime}\right),\left(\left[d_{2 j}^{\prime}\right], s_{2 j}^{\prime}\right)\right)$ and $P_{2}$ with $s k$ involve in the SMIN protocol, for $1 \leq j \leq\left\lfloor\frac{n u m}{2}\right\rfloor$. At the end of the first iteration, only $P_{1}$ knows $\left[\min \left(d_{2 j-1}^{\prime}, d_{2 j}^{\prime}\right)\right]$ and $s_{\min \left(d_{2 j-1}^{\prime}, d_{2 j}^{\prime}\right)}^{\prime}$, and nothing is revealed to $P_{2}$, for $1 \leq j \leq\left\lfloor\frac{n u m}{2}\right\rfloor$. Also, $P_{1}$ stores the result $\left[\min \left(d_{2 j-1}^{\prime}, d_{2 j}^{\prime}\right)\right]$ and $s_{\min \left(d_{2 j-1}^{\prime}, d_{2 j}^{\prime}\right)}^{\prime}$ in $\left[d_{2 j-1}^{\prime}\right]$ and $s_{2 j-1}^{\prime}$, respectively. In addition, $P_{1}$ updates the values of $\left[d_{2 j}^{\prime}\right], s_{2 j}^{\prime}$ to 0 and num to $\left\lceil\frac{\text { num }}{2}\right\rceil$, respectively.

During the $i^{t h}$ iteration, only the non-zero vectors (along with the corresponding encrypted secrets) are involved in SMIN, for $2 \leq i \leq\left\lceil\log _{2} n\right\rceil$. For example, during the second iteration (i.e., $\left.i=2\right)$, only $\left(\left[d_{1}^{\prime}\right], s_{1}^{\prime}\right),\left(\left[d_{3}^{\prime}\right], s_{3}^{\prime}\right)$, and so on are involved. Note that in each iteration, the output is revealed only to $P_{1}$ and num is updated to $\left\lceil\frac{\text { num }}{2}\right\rceil$. At the end of $\operatorname{SMIN}_{n}, P_{1}$ assigns the final encrypted binary vector of global minimum value, i.e., $\left[\min \left(d_{1}, \ldots, d_{n}\right)\right]$ which is stored in $\left[d_{1}^{\prime}\right]$, to $\left[d_{\min }\right]$. In addition, $P_{1}$ assigns $s_{1}^{\prime}$ to $E_{p k}\left(s_{d_{\min }}\right)$.

Example 6 Suppose $P_{1}$ holds $\left\langle\left[d_{1}\right], \ldots,\left[d_{6}\right]\right\rangle$ (i.e., $n=6$ ). For simplicity, here we are assuming that there are no secrets associated with $d_{i}$ 's. Then, based on the $S M I N_{n}$ protocol, the binary execution tree (in a bottom-up fashion) to compute $\left[\min \left(d_{1}, \ldots, d_{6}\right)\right]$ is shown in Figure $\square$ Note that, $\left[d_{i}^{\prime}\right]$ is initially set to $\left[d_{i}\right]$, for $1 \leq i \leq 6$.

Secure Bit-OR (SBOR). Suppose $P_{1}$ holds $\left(E_{p k}\left(o_{1}\right), E_{p k}\left(o_{2}\right)\right)$ and $P_{2}$ holds $s k$, where $o_{1}$ and $o_{2}$ are two bits not known to both parties. The goal of the SBOR protocol is to securely compute $E_{p k}\left(o_{1} \vee o_{2}\right)$. At the end of this protocol, only $P_{1}$ knows $E_{p k}\left(o_{1} \vee o_{2}\right)$. During this process, no information related to $o_{1}$ and $o_{2}$ is revealed to $P_{1}$ and $P_{2}$. Given the secure multiplication (SM) protocol, $P_{1}$ can compute $E_{p k}\left(o_{1} \vee o_{2}\right)$ as follows:

- $P_{1}$ with input $\left(E_{p k}\left(o_{1}\right), E_{p k}\left(o_{2}\right)\right)$ and $P_{2}$ involve in the SM protocol. At the end of this step, the output $E_{p k}\left(o_{1} * o_{2}\right)$ is known only to $P_{1}$. Note that, since $o_{1}$ and $o_{2}$ are bits, $E_{p k}\left(o_{1} * o_{2}\right)=E_{p k}\left(o_{1} \wedge o_{2}\right)$.

- $E_{p k}\left(o_{1} \vee o_{2}\right)=E_{p k}\left(o_{1}+o_{2}\right) * E_{p k}\left(o_{1} \wedge o_{2}\right)^{N-1}$. 
We emphasize that, for any given two bits $o_{1}$ and $o_{2}$, the property $o_{1} \vee o_{2}=o_{1}+o_{2}-o_{1} \wedge o_{2}$ always holds. Note that, by homomorphic addition property, $E_{p k}\left(o_{1}+o_{2}\right)=E_{p k}\left(o_{1}\right) * E_{p k}\left(o_{2}\right)$.

Secure Frequency (SF). Consider a situation where $P_{1}$ holds $\left(\left\langle E_{p k}\left(c_{1}\right), \ldots, E_{p k}\left(c_{w}\right)\right\rangle,\left\langle E_{p k}\left(c_{1}^{\prime}\right), \ldots, E_{p k}\left(c_{k}^{\prime}\right)\right\rangle\right)$ and $P_{2}$ holds the secret key $s k$. The goal of the SF protocol is to securely compute $E_{p k}\left(f\left(c_{j}\right)\right)$, for $1 \leq j \leq w$. Here $f\left(c_{j}\right)$ denotes the number of times element $c_{j}$ occurs (i.e., frequency) in the list $\left\langle c_{1}^{\prime}, \ldots, c_{k}^{\prime}\right\rangle$. We explicitly assume that $c_{i}^{\prime} \in\left\{c_{1}, \ldots, c_{w}\right\}$, for $1 \leq i \leq k$.

The output $\left\langle E_{p k}\left(f\left(c_{1}\right)\right), \ldots, E_{p k}\left(f\left(c_{w}\right)\right)\right\rangle$ is revealed only to $P_{1}$. During the SF protocol, neither $c_{i}^{\prime}$ nor $c_{j}$ is revealed to $P_{1}$ and $P_{2}$. Also, $f\left(c_{j}\right)$ is kept private from both $P_{1}$ and $P_{2}$, for $1 \leq i \leq k$ and $1 \leq j \leq w$.

The overall steps involved in the proposed SF protocol are shown in Algorithm 5 To start with, $P_{1}$ initially computes an encrypted vector $S_{i}$ such that $S_{i, j}=E_{p k}\left(c_{j}-c_{i}^{\prime}\right)$, for $1 \leq j \leq w$. Then, $P_{1}$ randomizes $S_{i}$ componentwise to get $S_{i, j}^{\prime}=E_{p k}\left(r_{i, j} *\left(c_{j}-c_{i}^{\prime}\right)\right)$, where $r_{i, j}$ is a random number in $\mathbb{Z}_{N}$. After this, for $1 \leq i \leq k, P_{1}$ randomly permutes $S_{i}^{\prime}$ component-wise using a random permutation function $\pi_{i}$ (known only to $P_{1}$ ). The output $Z_{i} \leftarrow \pi_{i}\left(S_{i}^{\prime}\right)$ is sent to $P_{2}$. Upon receiving, $P_{2}$ decrypts $Z_{i}$ component-wise, computes a vector $u_{i}$ and proceeds as follows:

- If $D_{s k}\left(Z_{i, j}\right)=0$, then $u_{i, j}$ is set to 1 . Otherwise, $u_{i, j}$ is set to 0 .

- The observation is, since $c_{i}^{\prime} \in\left\{c_{1}, \ldots, c_{w}\right\}$, that exactly one of the entries in vector $Z_{i}$ is an encryption of 0 and the rest are encryptions of random numbers. This further implies that exactly one of the decrypted values of $Z_{i}$ is 0 and the rest are random numbers. Precisely, if $u_{i, j}=1$, then $c_{i}^{\prime}=c_{\pi^{-1}(j)}$.

- Compute $U_{i, j}=E_{p k}\left(u_{i, j}\right)$ and send it to $P_{1}$, for $1 \leq i \leq k$ and $1 \leq j \leq w$.

Upon receiving $U, P_{1}$ performs row-wise inverse permutation on it to get $V_{i}=\pi_{i}^{-1}\left(U_{i}\right)$, for $1 \leq i \leq k$. Finally, $P_{1}$ computes $E_{p k}\left(c_{j}\right)=\prod_{i=1}^{k} V_{i, j}$ locally, for $1 \leq j \leq w$.

\section{Security Analysis of Privacy-Preserving Primitives under the Semi-Honest Model}

First of all, we emphasize that the outputs in the above mentioned protocols are always in encrypted format, and are known only to $P_{1}$. Also, all the intermediate results revealed to $P_{2}$ are either random or pseudo-random. Note that, the SBD protocol in [50] is secure under the semi-honest model. Therefore, here we provide security proofs for the other protocols under the semi-honest model. Informally speaking, we claim that all the intermediate results seen by $P_{1}$ and $P_{2}$ in the mentioned protocols are either random or pseudo-random.

As mentioned in Section 2.3, to formally prove that a protocol is secure [26] under the semi-honest model, we need to show that the simulated execution image of that protocol is computationally indistinguishable from its actual execution image. Remember that, an execution image generally includes the messages exchanged and the information computed from these messages.

\subsection{Proof of Security for SM}

According to Algorithm 11 let the execution image of $P_{2}$ be denoted by $\Pi_{P_{2}}(\mathrm{SM})$ which is given by $\Pi_{P_{2}}(\mathrm{SM})=$ $\left\{\left\langle a^{\prime}, h_{a}\right\rangle,\left\langle b^{\prime}, h_{b}\right\rangle\right\}$ where $h_{a}=a+r_{a} \bmod N$ and $h_{b}=b+r_{b} \bmod N$ are derived upon decrypting $a^{\prime}$ and $b^{\prime}$, respectively. Note that $h_{a}$ and $h_{b}$ are random numbers in $\mathbb{Z}_{N}$. Suppose the simulated image of $P_{2}$ be denoted by $\Pi_{P_{2}}^{S}(\mathrm{SM})$, where $\Pi_{P_{2}}^{S}(\mathrm{SM})=\left\{\left\langle a^{*}, r_{a}^{\prime}\right\rangle,\left\langle b^{*}, r_{b}^{\prime}\right\rangle\right\}$ Here $a^{*}$ and $b^{*}$ are randomly generated from $\mathbb{Z}_{N^{2}}$ whereas $r_{a}^{\prime}$ and $r_{b}^{\prime}$ are randomly generated from $\mathbb{Z}_{N}$. Since $E_{p k}$ is a semantically secure encryption scheme with resulting ciphertext size less than $N^{2}, a^{\prime}$ and $b^{\prime}$ are computationally indistinguishable from $a^{*}$ and $b^{*}$, respectively. Similarly, as $r_{a}$ and $r_{b}$ are randomly chosen from $\mathbb{Z}_{N}, h_{a}$ and $h_{b}$ are computationally indistinguishable from $r^{\prime} a$ and $r_{b}^{\prime}$, respectively. Combining the two results, we can conclude that $\Pi_{P_{2}}(\mathrm{SM})$ is computationally indistinguishable from $\Pi_{P_{2}}^{S}(\mathrm{SM})$.

Similarly, the execution image of $P_{1}$ in SM is given by $\Pi_{P_{1}}(\mathrm{SM})=\left\{h^{\prime}\right\}$. Here $h^{\prime}$ is an encrypted value. Let the simulated image of $P_{1}$ be given by $\Pi_{P_{1}}^{S}(\mathrm{SM})=\left\{h^{*}\right\}$, where $h^{*}$ is randomly chosen from $\mathbb{Z}_{N^{2}}$. Since $E_{p k}$ is a semantically secure encryption scheme with resulting ciphertext size less than $N^{2}, h^{\prime}$ is computationally indistinguishable 


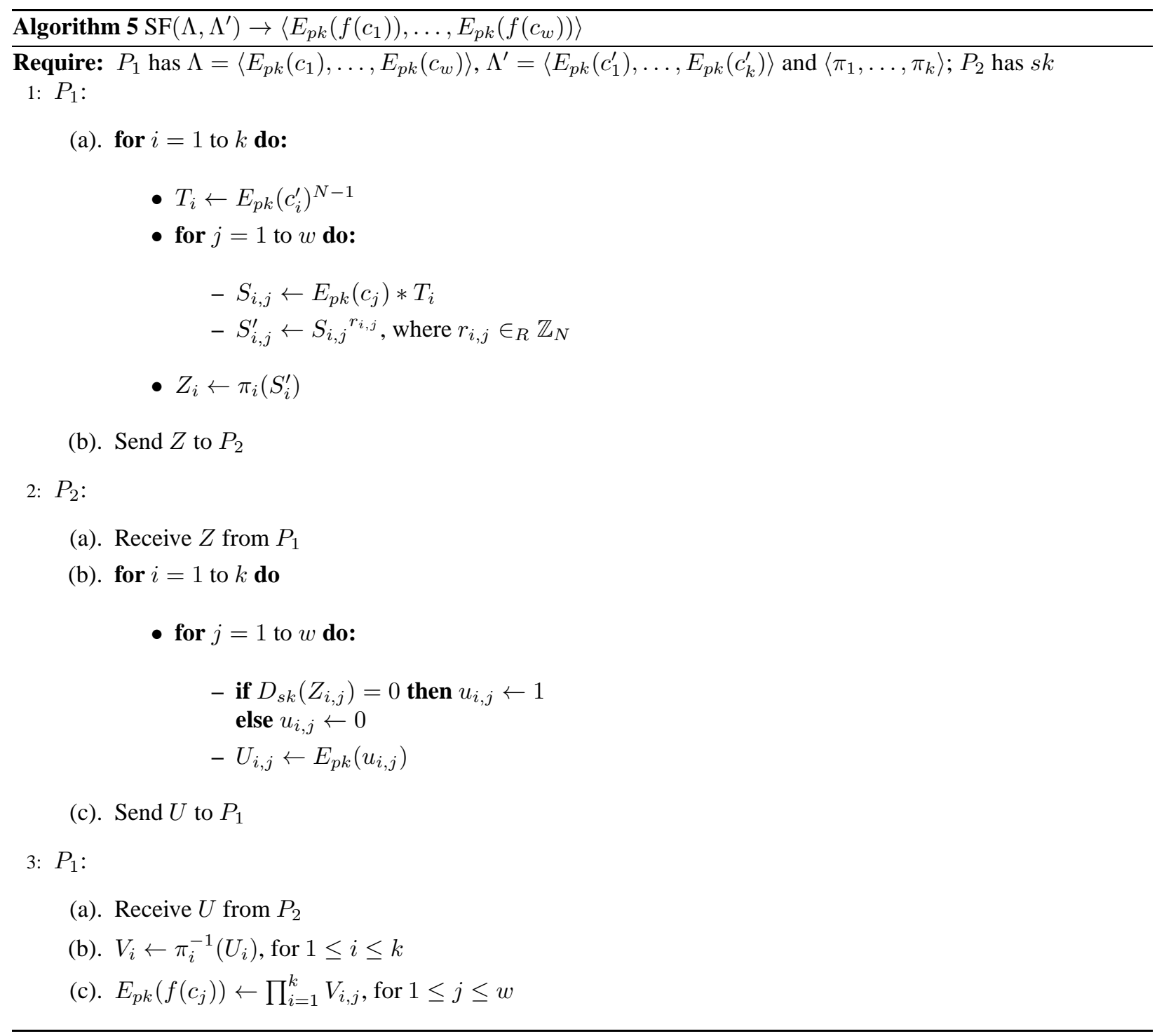

from $h^{*}$. As a result, $\Pi_{P_{1}}(\mathrm{SM})$ is computationally indistinguishable from $\Pi_{P_{1}}^{S}(\mathrm{SM})$. Putting the above results together and following from Definition 1, we can claim that SM is secure under the semi-honest model.

\subsection{Proof of Security for SSED}

The security of SSED directly follows from SM which is used as the fundamental building block in SSED. This is because, apart from SM, the rest of the steps in SSED are non-interactive. More specifically, as shown in Algorithm 2. $P_{1}$ and $P_{2}$ jointly compute $E_{p k}\left(\left(x_{i}-y_{i}\right)^{2}\right)$ using $\mathrm{SM}$, for $1 \leq i \leq m$. After this, $P_{1}$ performs homomorphic operations on $E_{p k}\left(\left(x_{i}-y_{i}\right)^{2}\right)$ locally (i.e., no interaction between $P_{1}$ and $\left.P_{2}\right)$.

\subsection{Proof of Security for SMIN}

According to Algorithm 3 , let the execution image of $P_{2}$ be denoted by $\Pi_{P_{2}}(\mathrm{SMIN})$, where

$$
\Pi_{P_{2}}(\mathrm{SMIN})=\left\{\langle\delta, s+\bar{r} \bmod N\rangle,\left\langle\Gamma_{i}^{\prime}, \mu_{i}+\hat{r}_{i} \bmod N\right\rangle,\left\langle L_{i}^{\prime}, \alpha\right\rangle\right\}
$$


Observe that $s+\bar{r} \bmod N$ and $\mu_{i}+\hat{r}_{i} \bmod N$ are derived upon decrypting $\delta$ and $\Gamma_{i}^{\prime}$, for $1 \leq i \leq l$, respectively. Note that the modulo operator is implicit in the decryption function. Also, $P_{2}$ receives $L^{\prime}$ from $P_{1}$ and let $\alpha$ denote the (oblivious) comparison result computed from $L^{\prime}$. Without loss of generality, suppose the simulated image of $P_{2}$ be $\Pi_{P_{2}}^{S}(\mathrm{SMIN})$, where

$$
\Pi_{P_{2}}^{S}(\mathrm{SMIN})=\left\{\left\langle\delta^{*}, r^{*}\right\rangle,\left\langle s_{1, i}^{\prime}, s_{2, i}^{\prime}\right\rangle,\left\langle s_{3, i}^{\prime}, \alpha^{\prime}\right\rangle \mid \text { for } 1 \leq i \leq l\right\}
$$

Here $\delta^{*}, s_{1, i}^{\prime}$ and $s_{3, i}^{\prime}$ are randomly generated from $\mathbb{Z}_{N^{2}}$ whereas $r^{*}$ and $s_{2, i}^{\prime}$ are randomly generated from $\mathbb{Z}_{N}$. In addition, $\alpha^{\prime}$ denotes a random bit. Since $E_{p k}$ is a semantically secure encryption scheme with resulting ciphertext size less than $N^{2}, \delta$ is computationally indistinguishable from $\delta^{*}$. Similarly, $\Gamma_{i}^{\prime}$ and $L_{i}^{\prime}$ are computationally indistinguishable from $s_{1, i}^{\prime}$ and $s_{3, i}^{\prime}$, respectively. Also, as $\bar{r}$ and $\hat{r}_{i}$ are randomly generated from $\mathbb{Z}_{N}, s+\bar{r} \bmod N$ and $\mu_{i}+\hat{r}_{i} \bmod N$ are computationally indistinguishable from $r^{*}$ and $s_{2, i}^{\prime}$, respectively. Furthermore, because the functionality is randomly chosen by $P_{1}$ (at step 1(a) of Algorithm 3), $\alpha$ is either 0 or 1 with equal probability. Thus, $\alpha$ is computationally indistinguishable from $\alpha^{\prime}$. Combining all these results together, we can conclude that $\Pi_{P_{2}}(\mathrm{SMIN})$ is computationally indistinguishable from $\Pi_{P_{2}}^{S}$ (SMIN) based on Definition 1. This implies that during the execution of SMIN, $P_{2}$ does not learn any information regarding $u, v, s_{u}, s_{v}$ and the actual comparison result. Intuitively speaking, the information $P_{2}$ has during an execution of SMIN is either random or pseudo-random, so this information does not disclose anything regarding $u, v, s_{u}$ and $s_{v}$. Additionally, as $F$ is known only to $P_{1}$, the actual comparison result is oblivious to $P_{2}$.

On the other hand, the execution image of $P_{1}$, denoted by $\Pi_{P_{1}}(\mathrm{SMIN})$, is given by

$$
\Pi_{P_{1}}(\mathrm{SMIN})=\left\{M_{i}^{\prime}, E_{p k}(\alpha), \delta^{\prime} \mid \text { for } 1 \leq i \leq l\right\}
$$

Here $M_{i}^{\prime}$ and $\delta^{\prime}$ are encrypted values, which are random in $\mathbb{Z}_{N^{2}}$, received from $P_{2}$ (at step 3(a) of Algorithm 3). Let the simulated image of $P_{1}$ be $\Pi_{P_{1}}^{S}(\mathrm{SMIN})$, where

$$
\Pi_{P_{1}}^{S}(\mathrm{SMIN})=\left\{s_{4, i}^{\prime}, b^{\prime}, b^{\prime \prime} \mid \text { for } 1 \leq i \leq l\right\}
$$

The values $s_{4, i}^{\prime}, b^{\prime}$ and $b^{\prime \prime}$ are randomly generated from $\mathbb{Z}_{N^{2}}$. Since $E_{p k}$ is a semantically secure encryption scheme with resulting ciphertext size less than $N^{2}$, it implies that $M_{i}^{\prime}, E_{p k}(\alpha)$ and $\delta^{\prime}$ are computationally indistinguishable from $s_{4, i}, b^{\prime}$ and $b^{\prime \prime}$, respectively. Therefore, $\Pi_{P_{1}}(\mathrm{SMIN})$ is computationally indistinguishable from $\Pi_{P_{1}}^{S}$ (SMIN) based on Definition 1. As a result, $P_{1}$ cannot learn any information regarding $u, v, s_{u}, s_{v}$ and the comparison result during the execution of SMIN.

Based on the above analysis, we can say that the proposed SMIN protocol is secure under the semi-honest model (following from Definition 1).

\subsection{Proof of Security for $\mathrm{SMIN}_{n}$}

According to Algorithm 4 it is clear that $\mathrm{SMIN}_{n}$ uses the SMIN protocol as a building block in an iterative manner. As proved above, SMIN is secure under the semi-honest model. Also, the output of SMIN which are passed as input to the next iteration in $\mathrm{SMIN}_{n}$ are in encrypted format. Note that, $\mathrm{SMIN}_{n}$ is solely based on SMIN and there are no other interactive steps between $P_{1}$ and $P_{2}$. Hence, by Composition Theorem [26], we claim that sequential combination of SMIN routines lead to our $\mathrm{SMIN}_{n}$ protocol that guarantees security under the semi-honest model.

\subsection{Proof of Security for SBOR}

The security of SBOR depends solely on the underlying SM protocol. This is because, the only step at which $P_{1}$ and $P_{2}$ interact in SBOR is during SM. Since SM is secure under the semi-honest model, we claim that SBOR is also secure under the semi-honest model. 


\subsection{Proof of Security for SF}

Without loss of generality, let the execution image of SF for $P_{2}$ be denoted by $\Pi_{P_{2}}(\mathrm{SF})$, and is given as (according to Algorithm 5)

$$
\Pi_{P_{2}}(\mathrm{SF})=\left\{Z_{i, j}, u_{i, j} \mid \text { for } 1 \leq j \leq w\right\}
$$

where $u_{i, j}$ is derived upon decrypting $Z_{i, j}$ (at step 2(b) of Algorithm 5). Suppose the simulated image of $P_{2}$ be denoted by $\Pi_{P_{2}}^{S}(\mathrm{SF})$ which can be given by

$$
\Pi_{P_{2}}^{S}(\mathrm{SF})=\left\{Z_{i, j}^{*}, u_{i, j}^{*} \mid \text { for } 1 \leq j \leq w\right\}
$$

Here $Z_{i, j}^{*}$ is randomly generated from $\mathbb{Z}_{N^{2}}$. Also, $u_{i}^{*}$ is a vector generated at random such that exactly one of them is 0 and the rest are random numbers in $\mathbb{Z}_{N}$. Since $E_{p k}$ is a semantically secure encryption scheme with resulting ciphertext size less than $N^{2}, Z_{i, j}$ is computationally indistinguishable from $Z_{i, j}^{*}$. Also, since $\pi_{i}$ is a random permutation function known only to $P_{1}, u_{i}$ will be a vector with exactly one zero (at random location) and the rest are random numbers in $\mathbb{Z}_{N}$. Hence, $u_{i}$ is computationally indistinguishable from $u_{i}^{*}$. Thus, we can claim that $\Pi_{P_{2}}(\mathrm{SF})$ is computationally indistinguishable from $\Pi_{P_{2}}^{S}(\mathrm{SF})$.

On the other hand, let the execution image of $P_{1}$ be denoted by $\Pi_{P_{1}}(\mathrm{SF})$, and is given by

$$
\Pi_{P_{1}}(\mathrm{SF})=\left\{U_{i, j} \mid \text { for } 1 \leq i \leq k \text { and } 1 \leq j \leq w\right\}
$$

Here $U_{i, j}$ is an encrypted value sent by $P_{2}$ at step 2(c) of Algorithm 5. Suppose the simulated image of $P_{1}$ be given by

$$
\Pi_{P_{1}}^{S}(\mathrm{SF})=\left\{U_{i, j}^{*} \mid \text { for } 1 \leq i \leq k \text { and } 1 \leq j \leq w\right\}
$$

where $U_{i, j}^{*}$ is a random number in $\mathbb{Z}_{N^{2}}$. Since $E_{p k}$ is a semantically secure encryption scheme with resulting ciphertext size less than $N^{2}, U_{i, j}$ is computationally indistinguishable from $U_{i, j}^{*}$. As a result, $\Pi_{P_{1}}(\mathrm{SF})$ is computationally indistinguishable from $\Pi_{P_{1}}^{S}(\mathrm{SF})$. Combining all the above results, we can claim that SF is secure under the semihonest model according on Definition 1.

\section{The Proposed Protocol}

In this section, we propose a novel privacy-preserving $k$-NN classification protocol, denoted by $\mathrm{PP} k \mathrm{NN}$, which is constructed using the protocols discussed in Section 3 as building blocks. As mentioned earlier, we assume that Alice's database consists of $n$ records, denoted by $D=\left\langle t_{1}, \ldots, t_{n}\right\rangle$, and $m+1$ attributes, where $t_{i, j}$ denotes the $j^{t h}$ attribute value of record $t_{i}$. Initially, Alice encrypts her database attribute-wise, that is, she computes $E_{p k}\left(t_{i, j}\right)$, for $1 \leq i \leq n$ and $1 \leq j \leq m+1$, where column $(m+1)$ contains the class labels. Let the encrypted database be denoted by $D^{\prime}$. We assume that Alice outsources $D^{\prime}$ as well as the future classification process to the cloud. Without loss of generality, we assume that all attribute values and their Euclidean distances lie in $\left[0,2^{l}\right)$. In addition, let $w$ denote the number of unique class labels in $D$.

In our problem setting, we assume the existence of two non-colluding semi-honest cloud service providers, denoted by $C_{1}$ and $C_{2}$, which together form a federated cloud. Under this setting, Alice outsources her encrypted database $D^{\prime}$ to $C_{1}$ and the secret key $s k$ to $C_{2}$. Here it is possible for the data owner Alice to replace $C_{2}$ with her private server. However, if Alice has a private server, we can argue that there is no need for data outsourcing from Alice's point of view. The main purpose of using $C_{2}$ can be motivated by the following two reasons. (i) With limited computing resource and technical expertise, it is in the best interest of Alice to completely outsource its data management and operational tasks to a cloud. For example, Alice may want to access her data and analytical results using a smart phone or any device with very limited computing capability. (ii) Suppose Bob wants to keep his input query and access patterns private from Alice. In this case, if Alice uses a private server, then she has to perform computations assumed by $C_{2}$ under which the very purpose of outsourcing the encrypted data to $C_{1}$ is negated.

In general, whether Alice uses a private server or cloud service provider $C_{2}$ actually depends on her resources. In particular to our problem setting, we prefer to use $C_{2}$ as this avoids the above mentioned disadvantages (i.e., in case 
of Alice using a private server) altogether. In our solution, after outsourcing encrypted data to the cloud, Alice does not participate in any future computations.

The goal of the PP $k N N$ protocol is to classify users' query records using $D^{\prime}$ in a privacy-preserving manner. Consider an authorized user Bob who wants to classify his query record $q=\left\langle q_{1}, \ldots, q_{m}\right\rangle$ based on $D^{\prime}$ in $C_{1}$. The proposed PP $k$ NN protocol mainly consists of the following two stages:

- Stage 1 - Secure Retrieval of $k$-Nearest Neighbors (SR $k N N)$ :

In this stage, Bob initially sends his query $q$ (in encrypted form) to $C_{1}$. After this, $C_{1}$ and $C_{2}$ involve in a set of sub-protocols to securely retrieve (in encrypted form) the class labels corresponding to the $k$-nearest neighbors of the input query $q$. At the end of this step, encrypted class labels of $k$-nearest neighbors are known only to $C_{1}$.

- Stage 2 - Secure Computation of Majority Class $\left(\mathrm{SCMC}_{k}\right)$ :

Following from Stage 1, $C_{1}$ and $C_{2}$ jointly compute the class label with a majority voting among the $k$-nearest neighbors of $q$. At the end of this step, only Bob knows the class label corresponding to his input query record $q$.

The main steps involved in the proposed $\mathrm{PP} k \mathrm{NN}$ protocol are as shown in Algorithm 6 . We now explain each of the two stages in PP $k \mathrm{NN}$ in detail.

\subsection{Stage 1 : Secure Retrieval of $k$-Nearest Neighbors ( $\mathbf{S R} k \mathbf{N N})$}

During Stage 1, Bob initially encrypts his query $q$ attribute-wise, that is, he computes $E_{p k}(q)=\left\langle E_{p k}\left(q_{1}\right), \ldots, E_{p k}\left(q_{m}\right)\right\rangle$ and sends it to $C_{1}$. The main steps involved in Stage 1 are shown as steps 1 to 3 in Algorithm 6 . Upon receiving $E_{p k}(q), C_{1}$ with private input $\left(E_{p k}(q), E_{p k}\left(t_{i}\right)\right)$ and $C_{2}$ with the secret key $s k$ jointly involve in the SSED protocol. Here $E_{p k}\left(t_{i}\right)=\left\langle E_{p k}\left(t_{i, 1}\right), \ldots, E_{p k}\left(t_{i, m}\right)\right\rangle$, for $1 \leq i \leq n$. The output of this step, denoted by $E_{p k}\left(d_{i}\right)$, is the encryption of squared Euclidean distance between $q$ and $t_{i}$, i.e., $d_{i}=\left|q-t_{i}\right|^{2}$. As mentioned earlier, $E_{p k}\left(d_{i}\right)$ is known only to $C_{1}$, for $1 \leq i \leq n$. We emphasize that the computation of exact Euclidean distance between encrypted vectors is hard to achieve as it involves square root. However, in our problem, it is sufficient to compare the squared Euclidean distances as it preserves relative ordering. Then, $C_{1}$ with input $E_{p k}\left(d_{i}\right)$ and $C_{2}$ securely compute the encryptions of the individual bits of $d_{i}$ using the SBD protocol. Note that the output $\left[d_{i}\right]=\left\langle E_{p k}\left(d_{i, 1}\right), \ldots, E_{p k}\left(d_{i, l}\right)\right\rangle$ is known only to $C_{1}$, where $d_{i, 1}$ and $d_{i, l}$ are the most and least significant bits of $d_{i}$, for $1 \leq i \leq n$, respectively.

After this, $C_{1}$ and $C_{2}$ compute the encryptions of class labels corresponding to the $k$-nearest neighbors of $q$ in an iterative manner. More specifically, they compute $E_{p k}\left(c_{1}^{\prime}\right)$ in the first iteration, $E_{p k}\left(c_{2}^{\prime}\right)$ in the second iteration, and so on. Here $c_{s}^{\prime}$ denotes the class label of $s^{\text {th }}$ nearest neighbor to $q$, for $1 \leq s \leq k$. At the end of $k$ iterations, only $C_{1}$ knows $\left\langle E_{p k}\left(c_{1}^{\prime}\right), \ldots, E_{p k}\left(c_{k}^{\prime}\right)\right\rangle$. To start with, consider the first iteration. $C_{1}$ and $C_{2}$ jointly compute the encryptions of the individual bits of the minimum value among $d_{1}, \ldots, d_{n}$ and encryptions of the location and class label corresponding to $d_{\min }$ using the $\operatorname{SMIN}_{n}$ protocol. That is, $C_{1}$ with input $\left(\theta_{1}, \ldots, \theta_{n}\right)$ and $C_{2}$ with sk compute $\left(\left[d_{\min }\right], E_{p k}(I), E_{p k}\left(c^{\prime}\right)\right)$, where $\theta_{i}=\left(\left[d_{i}\right], E_{p k}\left(I_{t_{i}}\right), E_{p k}\left(t_{i, m+1}\right)\right)$, for $1 \leq i \leq n$. Here $d_{\text {min }}$ denotes the minimum value among $d_{1}, \ldots, d_{n} ; I_{t_{i}}$ and $t_{i, m+1}$ denote the unique identifier and class label corresponding to the data record $t_{i}$, respectively. Specifically, $\left(I_{t_{i}}, t_{i, m+1}\right)$ is the secret information associated with $t_{i}$. For simplicity, this paper assumes $I_{t_{i}}=i$. In the output, $I$ and $c^{\prime}$ denote the index and class label corresponding to $d_{\min }$. The output ( $\left.\left[d_{\min }\right], E_{p k}(I), E_{p k}(c)\right)$ is known only to $C_{1}$. Now, $C_{1}$ performs the following operations locally:

- Assign $E_{p k}\left(c^{\prime}\right)$ to $E_{p k}\left(c_{1}^{\prime}\right)$. Remember that, according to the $\operatorname{SMIN}_{n}$ protocol, $c^{\prime}$ is equivalent to the class label of the data record that corresponds to $d_{\min }$. Thus, it is same as the class label of the most nearest neighbor to $q$.

- Compute the encryption of difference between $I$ and $i$, where $1 \leq i \leq n$. That is, $C_{1}$ computes $\tau_{i}=E_{p k}(i) *$ $E_{p k}(I)^{N-1}=E_{p k}(i-I)$, for $1 \leq i \leq n$.

- Randomize $\tau_{i}$ to get $\tau_{i}^{\prime}=\tau_{i}^{r_{i}}=E_{p k}\left(r_{i} *(i-I)\right)$, where $r_{i}$ is a random number in $\mathbb{Z}_{N}$. Note that $\tau_{i}^{\prime}$ is an encryption of either 0 or a random number, for $1 \leq i \leq n$. Also, it is worth noting that exactly one of the entries in $\tau^{\prime}$ is an encryption of 0 (which happens iff $i=I$ ) and the rest are encryptions of random numbers. Permute $\tau^{\prime}$ using a random permutation function $\pi$ (known only to $C_{1}$ ) to get $\beta=\pi\left(\tau^{\prime}\right)$ and send it to $C_{2}$. 


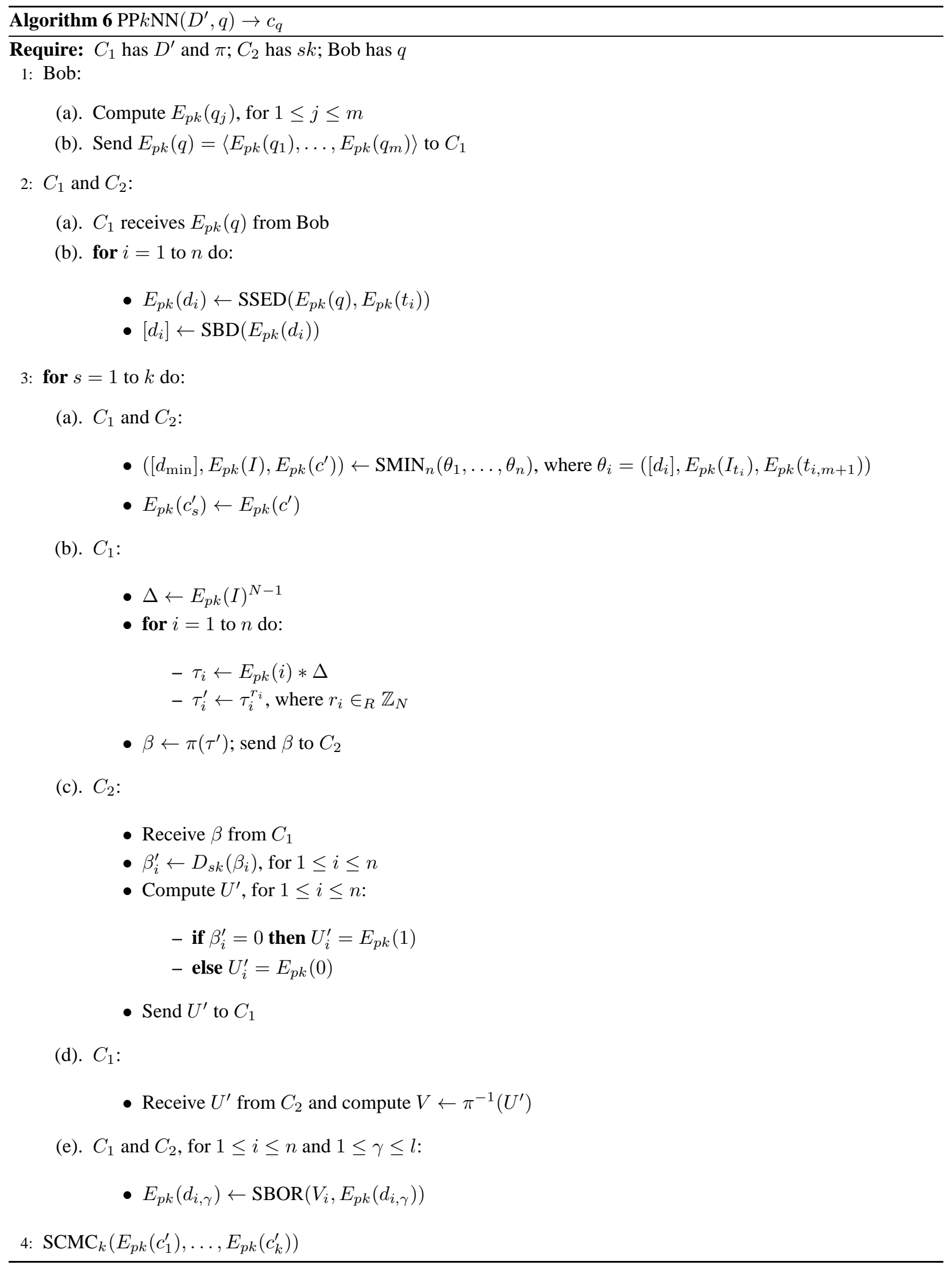


Upon receiving $\beta, C_{2}$ decrypts it component-wise to get $\beta_{i}^{\prime}=D_{s k}\left(\beta_{i}\right)$, for $1 \leq i \leq n$. After this, he/she computes an encrypted vector $U^{\prime}$ of length $n$ such that $U_{i}=E_{p k}(1)$ if $\beta_{i}^{\prime}=0$, and $E_{p k}(0)$ otherwise. Since exactly one of entries in $\tau^{\prime}$ is an encryption of 0 , this further implies that exactly one of the entries in $U^{\prime}$ is an encryption of 1 and the rest of them are encryptions of 0's. It is important to note that if $\beta_{k}^{\prime}=0$, then $\pi^{-1}(k)$ is the index of the data record that corresponds to $d_{\min }$. Then, $C_{2}$ sends $U^{\prime}$ to $C_{1}$. After receiving $U^{\prime}, C_{1}$ performs inverse permutation on it to get $V=\pi^{-1}\left(U^{\prime}\right)$. Note that exactly one of the entry in $V$ is $E_{p k}(1)$ and the remaining are encryptions of 0 's. In addition, if $V_{i}=E_{p k}(1)$, then $t_{i}$ is the most nearest tuple to $q$. However, $C_{1}$ and $C_{2}$ do not know which entry in $V$ corresponds to $E_{p k}(1)$.

Finally, $C_{1}$ updates the distance vectors $\left[d_{i}\right]$ due to the following reason:

- It is important to note that the first nearest tuple to $q$ should be obliviously excluded from further computations. However, since $C_{1}$ does not know the record corresponding to $E_{p k}\left(c_{1}^{\prime}\right)$, we need to obliviously eliminate the possibility of choosing this record again in next iterations. For this, $C_{1}$ obliviously updates the distance corresponding to $E_{p k}\left(c_{1}^{\prime}\right)$ to the maximum value, i.e., $2^{l}-1$. More specifically, $C_{1}$ updates the distance vectors with the help of $C_{2}$ using the SBOR protocol as below, for $1 \leq i \leq n$ and $1 \leq \gamma \leq l$.

$$
E_{p k}\left(d_{i, \gamma}\right)=\operatorname{SBOR}\left(V_{i}, E_{p k}\left(d_{i, \gamma}\right)\right)
$$

Note that when $V_{i}=E_{p k}(1)$, the corresponding distance vector $d_{i}$ is set to the maximum value. That is, under this case, $\left[d_{i}\right]=\left\langle E_{p k}(1), \ldots, E_{p k}(1)\right\rangle$. On the other hand, when $V_{i}=E_{p k}(0)$, the OR operation has no effect on the corresponding encrypted distance vector.

The above process is repeated until $k$ iterations, and in each iteration $\left[d_{i}\right]$ corresponding to the current chosen label is set to the maximum value. However, $C_{1}$ and $C_{2}$ do not know which $\left[d_{i}\right]$ is updated. In iteration $s, E_{p k}\left(c_{s}^{\prime}\right)$ is returned only to $C_{1}$. At the end of Stage $1, C_{1}$ has $\left\langle E_{p k}\left(c_{1}^{\prime}\right), \ldots, E_{p k}\left(c_{k}^{\prime}\right)\right\rangle$ - the list of encrypted class labels of $k$-nearest neighbors to the input query $q$.

\subsection{Stage 2 : Secure Computation of Majority Class $\left(\mathrm{SCMC}_{k}\right)$}

Without loss of generality, suppose Alice's dataset $D$ consists of $w$ unique class labels denoted by $c=\left\langle c_{1}, \ldots, c_{w}\right\rangle$. We assume that Alice outsources her list of encrypted classes to $C_{1}$. That is, Alice outsources $\left\langle E_{p k}\left(c_{1}\right), \ldots, E_{p k}\left(c_{w}\right)\right\rangle$ to $C_{1}$ along with her encrypted database $D^{\prime}$ during the data outsourcing step. Note that, for security reasons, Alice may add dummy categories into the list to protect the number of class labels, i.e., $w$ from $C_{1}$ and $C_{2}$. However, for simplicity, we assume that Alice does not add any dummy categories to $c$.

During Stage 2, $C_{1}$ with private inputs $\Lambda=\left\langle E_{p k}\left(c_{1}\right), \ldots, E_{p k}\left(c_{w}\right)\right\rangle$ and $\Lambda^{\prime}=\left\langle E_{p k}\left(c_{1}^{\prime}\right), \ldots, E_{p k}\left(c_{k}^{\prime}\right)\right\rangle$, and $C_{2}$ with $s k$ securely compute $E_{p k}\left(c_{q}\right)$. Here $c_{q}$ denotes the majority class label among $c_{1}^{\prime}, \ldots, c_{k}^{\prime}$. At the end of stage 2, only Bob knows the class label $c_{q}$.

The overall steps involved in Stage 2 are shown in Algorithm 7 To start with, $C_{1}$ and $C_{2}$ jointly compute the encrypted frequencies of each class label using the $k$-nearest set as input. That is, they compute $E_{p k}\left(f\left(c_{i}\right)\right)$ using $\left(\Lambda, \Lambda^{\prime}\right)$ as $C_{1}$ 's input to the secure frequency (SF) protocol, for $1 \leq i \leq w$. The output $\left\langle E_{p k}\left(f\left(c_{1}\right)\right), \ldots, E_{p k}\left(f\left(c_{w}\right)\right)\right\rangle$ is known only to $C_{1}$. Then, $C_{1}$ with $E_{p k}\left(f\left(c_{i}\right)\right)$ and $C_{2}$ with $s k$ involve in the secure bit-decomposition (SBD) protocol to compute $\left[f\left(c_{i}\right)\right]$, that is, vector of encryptions of the individual bits of $f\left(c_{i}\right)$, for $1 \leq i \leq w$. After this, $C_{1}$ and $C_{2}$ jointly involve in the $\mathrm{SMAX}_{w}$ protocol. Briefly, $\operatorname{SMAX}_{w}$ utilizes the sub-routine SMAX to eventually compute $\left(\left[f_{\max }\right], E_{p k}\left(c_{q}\right)\right)$ in an iterative fashion. Here $\left[f_{\max }\right]=\left[\max \left(f\left(c_{1}\right), \ldots, f\left(c_{w}\right)\right)\right]$ and $c_{q}$ denotes the majority class out of $\Lambda^{\prime}$. At the end, the output $\left(\left[f_{\max }\right], E_{p k}\left(c_{q}\right)\right)$ is known only to $C_{1}$. After this, $C_{1}$ computes $\gamma_{q}=E_{p k}\left(c_{q}+r_{q}\right)$, where $r_{q}$ is a random number in $\mathbb{Z}_{N}$ known only to $C_{1}$. Then, $C_{1}$ sends $\gamma_{q}$ to $C_{2}$ and $r_{q}$ to Bob. Upon receiving $\gamma_{q}$, $C_{2}$ decrypts it to get the randomized majority class label $\gamma_{q}^{\prime}=D_{s k}\left(\gamma_{q}\right)$ and sends it to Bob. Finally, upon receiving $r_{q}$ from $C_{1}$ and $\gamma_{q}^{\prime}$ from $C_{2}$, Bob computes the output class label corresponding to $q$ as $c_{q}=\gamma_{q}^{\prime}-r_{q} \bmod N$.

\subsection{Security Analysis of $P P k N N$ under the Semi-honest Model}

Here we provide a formal security proof for the proposed PP $k N N$ protocol under the semi-honest model. First of all, we stress that due to the encryption of $q$ and by semantic security of the Paillier cryptosystem, Bob's input query $q$ is 


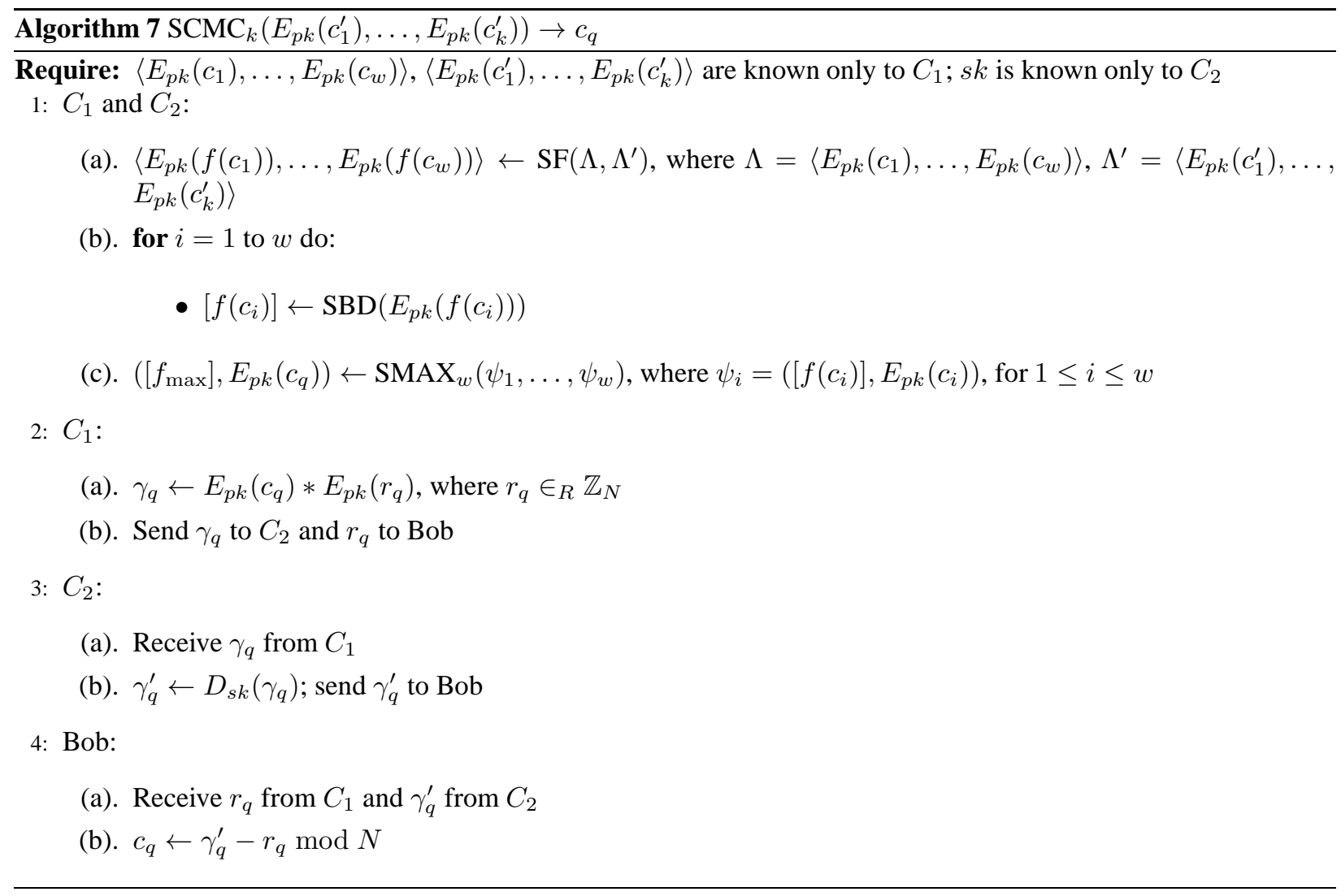

protected from Alice, $C_{1}$ and $C_{2}$ in our PP $k \mathrm{NN}$ protocol. Apart from guaranteeing query privacy, remember that the goal of $\mathrm{PP} k \mathrm{NN}$ is to protect data confidentiality and hide data access patterns.

In this paper, to prove a protocol's security under the semi-honest model, we adopted the well-known security definitions from the literature of secure multiparty computation (SMC). More specifically, as mentioned in Section 2.3. we adopt the security proofs based on the standard simulation paradigm [26]. For presentation purpose, we provide formal security proofs (under the semi-honest model) for Stages 1 and 2 of PP $k$ NN separately. Note that the outputs returned by each sub-protocol are in encrypted form and known only to $C_{1}$.

\subsubsection{Proof of Security for Stage 1}

As mentioned earlier, the computations involved in Stage 1 of PP $k N N$ are given as steps 1 to 3 in Algorithm 6 For ease of presentation, we consider the messages exchanged between $C_{1}$ and $C_{2}$ in a single iteration (however, similar analysis can be deduced for other iterations).

According to Algorithm 6 , the execution image of $C_{2}$ is given by

$$
\Pi_{C_{2}}(\mathrm{PP} k \mathrm{NN})=\left\{\left\langle\beta_{i}, \beta_{i}^{\prime}\right\rangle \mid \text { for } 1 \leq i \leq n\right\}
$$

where $\beta_{i}$ is an encrypted value which is random in $\mathbb{Z}_{N^{2}}$. Also, $\beta_{i}^{\prime}$ is derived upon decrypting $\beta_{i}$ by $C_{2}$. Remember that, exactly one of the entries in $\beta^{\prime}$ is 0 and the rest are random numbers in $\mathbb{Z}_{N}$. Without loss of generality, let the simulated image of $C_{2}$ be denoted by $\Pi_{C_{2}}^{S}(\mathrm{PP} k \mathrm{NN})$ and is given as

$$
\Pi_{C_{2}}^{S}(\mathrm{PP} k \mathrm{NN})=\left\{\left\langle a_{1, i}^{\prime}, a_{2, i}^{\prime}\right\rangle \mid \text { for } 1 \leq i \leq n\right\}
$$

here $a_{1, i}^{\prime}$ is randomly generated from $\mathbb{Z}_{N^{2}}$ and the vector $a_{2}^{\prime}$ is randomly generated in such a way that exactly one of the entries is 0 and the rest are random numbers in $\mathbb{Z}_{N}$. Since $E_{p k}$ is a semantically secure encryption scheme with 
resulting ciphertext size less than $\mathbb{Z}_{N^{2}}$, we claim that $\beta_{i}$ is computationally indistinguishable from $a_{1, i}^{\prime}$. In addition, since the random permutation function $\pi$ is known only to $C_{1}, \beta^{\prime}$ is a random vector of exactly one 0 and random numbers in $\mathbb{Z}_{N}$. Thus, $\beta^{\prime}$ is computationally indistinguishable from $a_{2}^{\prime}$. By combining the above results, we can conclude that $\Pi_{C_{2}}(\mathrm{PP} k \mathrm{NN})$ is computationally indistinguishable from $\Pi_{C_{2}}^{S}(\mathrm{PP} k \mathrm{NN})$. This implies that $C_{2}$ does not learn anything during the execution of Stage 1 in $\mathrm{PP} k \mathrm{NN}$.

On the other hand, suppose the execution image of $C_{1}$ be denoted by $\Pi_{C_{1}}(\mathrm{PP} k \mathrm{NN})$, and is given by

$$
\Pi_{C_{1}}(\mathrm{PP} k \mathrm{NN})=\left\{U^{\prime}\right\}
$$

where $U^{\prime}$ is an encrypted value sent by $C_{2}$ (at step 3(c) of Algorithm 6 ). Let the simulated image of $C_{1}$ in Stage 1 be denoted by $\Pi_{C_{1}}^{S}(\mathrm{PP} k \mathrm{NN})$, which is given as

$$
\Pi_{C_{1}}^{S}(\mathrm{PP} k \mathrm{NN})=\left\{a^{\prime}\right\}
$$

The value of $a^{\prime}$ is randomly generated from $\mathbb{Z}_{N^{2}}$. Since $E_{p k}$ is a semantically secure encryption scheme with resulting ciphertexts in $\mathbb{Z}_{N^{2}}$, we claim that $U^{\prime}$ is computationally indistinguishable from $a^{\prime}$. This implies that $\Pi_{C_{1}}(\mathrm{PP} k \mathrm{NN})$ is computationally indistinguishable from $\Pi_{C_{1}}^{S}(\mathrm{PP} k \mathrm{NN})$. Hence, $C_{1}$ cannot learn anything during the execution of Stage 1 in PP $k N N$. Combining all these results together, it is clear that Stage 1 of PP $k \mathrm{NN}$ is secure under the semi-honest model.

In each iteration, it is worth pointing out that $C_{1}$ and $C_{2}$ do not know which data record belongs to current global minimum. Thus, data access patterns are protected from both $C_{1}$ and $C_{2}$. Informally speaking, at step 3(c) of Algorithm 6, a component-wise decryption of $\beta$ reveals the tuple that satisfy the current global minimum distance to $C_{2}$. However, due to the random permutation by $C_{1}, C_{2}$ cannot trace back to the corresponding data record. Also, note that decryption operations on vector $\beta$ by $C_{2}$ will result in exactly one 0 and the rest of the results are random numbers in $\mathbb{Z}_{N}$. Similarly, since $U^{\prime}$ is an encrypted vector, $C_{1}$ cannot know which tuple corresponds to current global minimum distance.

\subsubsection{Security Proof for Stage 2}

In a similar fashion, we can formally prove that Stage 2 of $\mathrm{PP} k \mathrm{NN}$ is secure under the semi-honest model. Briefly, since the sub-protocols SF, SBD, and $\mathrm{SMAX}_{w}$ are secure, no information is revealed to $C_{2}$. On the other hand, the operations performed by $C_{1}$ are entirely on encrypted data; therefore, no information is revealed to $C_{1}$.

Furthermore, the output data of Stage 1 which are passed as input to Stage 2 are in encrypted format. Therefore, the sequential composition of the two stages lead to our PP $k \mathrm{NN}$ protocol and we claim it to be secure under the semihonest model according to the Composition Theorem [26]. In particular, based on the above discussions, it is clear that the proposed PP $k N N$ protocol protects the confidentiality of the data, user's input query, and also hides data access patterns from Alice, $C_{1}$, and $C_{2}$. Note that Alice does not participate in any computations of PPkNN.

\subsection{Security under the Malicious model}

The next step is to extend our PP $k \mathrm{NN}$ protocol into a secure protocol under the malicious model. Under the malicious model, an adversary (i.e., either $C_{1}$ or $C_{2}$ ) can arbitrarily deviate from the protocol to gain some advantage (e.g., learning additional information about inputs) over the other party. The deviations include, as an example, for $C_{1}$ (acting as a malicious adversary) to instantiate the $\mathrm{PP} k \mathrm{NN}$ protocol with modified inputs (say $E_{p k}\left(q^{\prime}\right)$ and $E_{p k}\left(t_{i}^{\prime}\right)$ ) and to abort the protocol after gaining partial information. However, in $\mathrm{PP} k \mathrm{NN}$, it is worth pointing out that neither $C_{1}$ nor $C_{2}$ knows the results of Stages 1 and 2. In addition, all the intermediate results are either random or pseudo-random values. Thus, even when an adversary modifies the intermediate computations he/she cannot gain any additional information. Nevertheless, as mentioned above, the adversary can change the intermediate data or perform computations incorrectly before sending them to the honest party which may eventually result in the wrong output. Therefore, we need to ensure that all the computations performed and messages sent by each party are correct.

Remember that the main goal of SMC is to ensure the honest parties to get the correct result and to protect their private input data from the malicious parties. Therefore, under the two-party SMC scenario, if both parties are malicious, there is no point to develop or adopt an SMC protocol at the first place. In the literature of SMC [14], 
it is the norm that at most one party can be malicious under the two-party scenario. When only one of the party is malicious, the standard way of preventing the malicious party from misbehaving is to let the honest party validate the other party's work using zero-knowledge proofs [11]. However, checking the validity of computations at each step of $\mathrm{PP} k \mathrm{NN}$ can significantly increase the overall cost.

An alternative approach, as proposed in [36], is to instantiate two independent executions of the PPkNN protocol by swapping the roles of the two parties in each execution. At the end of the individual executions, each party receives the output in encrypted form. This is followed by an equality test on their outputs. More specifically, suppose $E_{p k_{1}}\left(c_{q, 1}\right)$ and $E_{p k_{2}}\left(c_{q, 2}\right)$ be the outputs received by $C_{1}$ and $C_{2}$ respectively, where $p k_{1}$ and $p k_{2}$ are their respective public keys. Note that the outputs in our case are in encrypted format and the corresponding ciphertexts (resulted from the two executions) are under two different public key domains. Therefore, we stress that the equality test based on the additive homomorphic encryption properties which was used in [36] is not applicable to our problem. Nevertheless, $C_{1}$ and $C_{2}$ can perform the equality test based on the traditional garbled-circuit technique [35].

\subsection{Complexity Analysis}

The computation complexity of Stage 1 in PP $k$ NN is bounded by $O(n)$ instantiations of SBD and SSED, $O(k)$ instantiations of $\mathrm{SMIN}_{n}$, and $O(n * k * l)$ instantiations of SBOR. We emphasize that the computation complexity of the SBD protocol proposed in [50] is bounded by $O(l)$ encryptions and $O(l)$ exponentiations (under the assumption that encryption and decryption operations based on Paillier cryptosystem take similar amount of time). Also, the computation complexity of SSED is bounded by $O(m)$ encryptions and $O(m)$ exponentiations. In addition, the computation complexity of $\mathrm{SMIN}_{n}$ is bounded by $O\left(l * n * \log _{2} n\right)$ encryptions and $O\left(l * n * \log _{2} n\right)$ exponentiations. Since SBOR utilizes SM as a sub-routine, the computation cost of SBOR is bounded by (small) constant number of encryptions and exponentiations. Based on the above analysis, the total computation complexity of Stage 1 is bounded by $O\left(n *\left(l+m+k * l * \log _{2} n\right)\right)$ encryptions and exponentiations.

On the other hand, the computation complexity of Stage 2 is bounded by $O(w)$ instantiations of SBD, and one

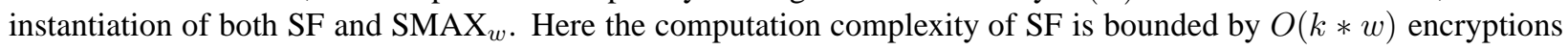
and $O(k * w)$ exponentiations. Therefore, the total computation complexity of Stage 2 is bounded by $O(w *(l+k+$ $\left.\left.l * \log _{2} w\right)\right)$ encryptions and exponentiations.

In general, $w \ll n$, therefore, the computation cost of Stage 1 should be significantly higher than that of Stage 2. This observation is further justified by our empirical results given in the next section.

\section{Empirical Results}

In this section, we discuss some experiments demonstrating the performance of our PP $k \mathrm{NN}$ protocol under different parameter settings. We used the Paillier cryptosystem [45] as the underlying additive homomorphic encryption scheme and implemented the proposed $\mathrm{PP} k \mathrm{NN}$ protocol in C. Various experiments were conducted on a Linux machine with an Intel $\AA$ Xeon ${ }^{\circledR}$ Six-Core ${ }^{\mathrm{TM}}$ CPU $3.07 \mathrm{GHz}$ processor and 12GB RAM running Ubuntu 12.04 LTS.

To the best of our knowledge, our work is the first effort to develop a secure $k$-NN classifier under the semi-honest model. Thus, there is no existing work to compare with our approach. Therefore, we evaluate the performance of our $\mathrm{PP} k \mathrm{NN}$ protocol under different parameter settings.

\subsection{Dataset and Experimental Setup}

For our experiments, we used the Car Evaluation dataset from the UCI KDD archive [9]. The dataset consists of 1728 data records (i.e., $n=1728$ ) with 6 input attributes (i.e., $m=6$ ). Also, there is a separate class attribute and the dataset is categorized into four different classes (i.e., $w=4$ ). We encrypted this dataset attribute-wise, using the Paillier encryption whose key size is varied in our experiments, and the encrypted data were stored on our machine. Based on our PP $k \mathrm{NN}$ protocol, we then executed a random query over this encrypted data. For the rest of this section, we do not discuss about the performance of Alice since it is a one-time cost. Instead, we evaluate and analyze the performances of the two stages in $\mathrm{PP} k \mathrm{NN}$ separately. 


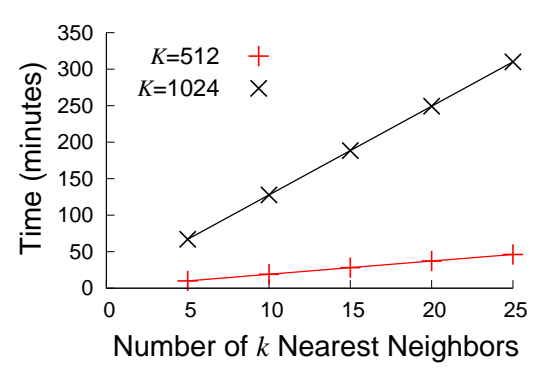

(a) Total cost of Stage 1

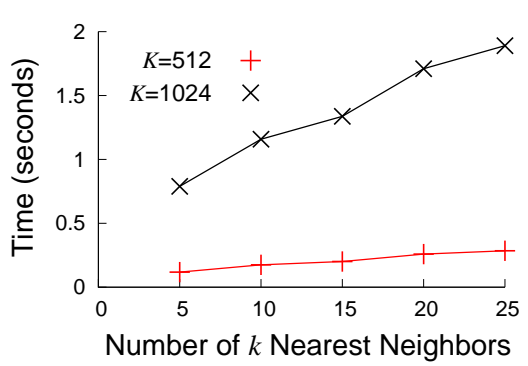

(b) Total cost of Stage 2

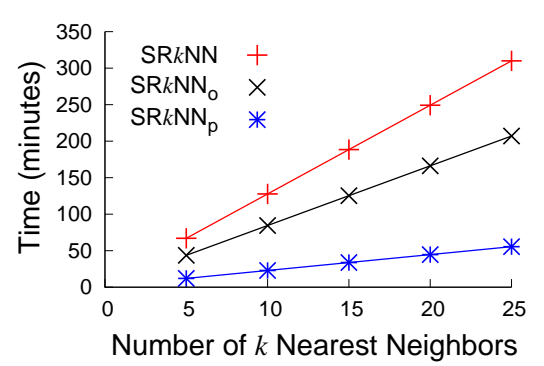

(c) Efficiency gains of Stage 1 for $K=1024$

Figure 2: Computation costs of PP $k \mathrm{NN}$ for varying number of $k$ nearest neighbors and different encryption key sizes in bits $(K)$

\subsection{Performance of $\mathbf{P P} k \mathbf{N N}$}

We first evaluated the computation costs of Stage 1 in $\mathrm{PP} k \mathrm{NN}$ for varying number of $k$-nearest neighbors. Also, the Paillier encryption key size $K$ is either 512 or 1024 bits. The results are shown in Figure 2(a) For $K=512$ bits, the computation cost of Stage 1 varies from 9.98 to 46.16 minutes when $k$ is changed from 5 to 25 , respectively. On the other hand, when $K=1024$ bits, the computation cost of Stage 1 varies from 66.97 to 309.98 minutes when $k$ is changed from 5 to 25 , respectively. In either case, we observed that the cost of Stage 1 grows almost linearly with $k$. In addition, for any given $k$, we identified that the cost of Stage 1 increases by almost a factor of 7 whenever $K$ is doubled. For example, when $k=10$, Stage 1 took 19.06 and 127.72 minutes to generate the encrypted class labels of the 10 nearest neighbors under $K=512$ and 1024 bits, respectively. Furthermore, when $k=5$, we observe that around $66.29 \%$ of cost in Stage 1 is accounted due to $\mathrm{SMIN}_{n}$ which is initiated $k$ times in PP $k \mathrm{NN}$ (once in each iteration). Also, the cost incurred due to $\mathrm{SMIN}_{n}$ increases from $66.29 \%$ to $71.66 \%$ when $k$ is increased from 5 to 25 .

We now evaluate the computation costs of Stage 2 for varying $k$ and $K$. As shown in Figure 2 (b) for $K=512$ bits, the computation time for Stage 2 to generate the final class label corresponding to the input query varies from 0.118 to 0.285 seconds when $k$ is changed from 5 to 25 . On the other hand, for $K=1024$ bits, Stage 2 took 0.789 and 1.89 seconds when $k=5$ and 25, respectively. The low computation costs of Stage 2 were due to $\mathrm{SMAX}_{w}$ which incurs significantly less computations than $\mathrm{SMIN}_{n}$ in Stage 1. This further justifies our theoretical analysis in Section 5.5. Note that, in our dataset, $w=4$ and $n=1728$. Like in Stage 1, for any given $k$, the computation time of Stage 2 increases by almost a factor of 7 whenever $K$ is doubled. E.g., when $k=10$, the computation time of Stage 2 varies from 0.175 to 1.158 seconds when the encryption key size $K$ is changed from 512 to 1024 bits. As shown in Figure 2(b), a similar analysis can be observed for other values of $k$ and $K$.

Based on the above results, it is clear that the computation cost of Stage 1 is significantly higher than that of Stage 2 in PP $k$ NN. Specifically, we observed that the computation time of Stage 1 accounts for at least $99 \%$ of the total time in PP $k$ NN. For example, when $k=10$ and $K=512$ bits, the computation costs of Stage 1 and 2 are 19.06 minutes and 0.175 seconds, respectively. Under this scenario, cost of Stage 1 is $99.98 \%$ of the total cost of PP $k N$. We also observed that the total computation time of $\mathrm{PP} k \mathrm{NN}$ grows almost linearly with $n$ and $k$.

\subsection{Performance Improvement of $\mathrm{PP} k \mathrm{NN}$}

We now discuss two different ways to boost the efficiency of Stage 1 (as the performance of PP $k N$ depends primarily on Stage 1) and empirically analyze their efficiency gains. First, we observe that some of the computations in Stage 1 can be pre-computed. For example, encryptions of random numbers, 0s and $1 \mathrm{~s}$ can be pre-computed (by the corresponding parties) in the offline phase. As a result, the online computation cost of Stage 1 (denoted by $\mathrm{SR}_{k} \mathrm{NN}_{\mathrm{o}}$ ) is expected to be improved. To see the actual efficiency gains of such a strategy, we computed the costs of $\mathrm{SR}_{k \mathrm{NN}}$ and compared them with the costs of Stage 1 without an offline phase (simply denoted by SR $k N$ ) and the results for $K=1024$ bits are shown in Figure 2(c). Irrespective of the values of $k$, we observed that $\mathrm{SR}_{k \mathrm{NN}}$ is around $33 \%$ faster than SR $k$ NN. E.g., when $k=10$, the computation costs of $\mathrm{SR} k \mathrm{NN}_{\mathrm{o}}$ and $\mathrm{SR} k \mathrm{NN}$ are 84.47 and 127.72 minutes, respectively (boosting the online running time of Stage 1 by $33.86 \%$ ). 


\begin{tabular}{|l|c|c|}
\hline Stage & Communication Size (in MBytes) & Network Delay (in seconds) \\
\hline Stage 1 & 154.741 & 123.79 \\
\hline Stage 2 & 0.037 & 0.0296 \\
\hline
\end{tabular}

Table 3: Communication sizes and network delays in PP $k$ NN for $k=10$ and $K=1024$ bits

Our second approach to improve the performance of Stage 1 is by using parallelism. Since operations on data records are independent of one another, we claim that most computations in Stage 1 can be parallelized. To empirically evaluate this claim, we implemented a parallel version of Stage 1 (denoted by $\mathrm{SR} k \mathrm{NN}_{\mathrm{p}}$ ) using OpenMP programming and compared its cost with the costs of SR $k$ NN (i.e., the serial version of Stage 1). The results for $K=1024$ bits are shown in Figure 2(c) The computation cost of $\mathrm{SR} k \mathrm{NN}_{\mathrm{p}}$ varies from 12.02 to 55.5 minutes when $k$ is changed from 5 to 25. We observe that $\mathrm{SR} k \mathrm{NN}_{\mathrm{p}}$ is almost 6 times more efficient than $\mathrm{SR} k \mathrm{NN}$. This is because our machine has 6 cores and thus computations can be run in parallel on 6 separate threads. Based on the above discussions, it is clear that efficiency of Stage 1 can indeed be improved significantly using parallelism. Moreover, we can also use the existing map-reduce techniques to execute parallel operations on multiple nodes to drastically improve the performance further. Hence, the level of achievable performance in PP $k \mathrm{NN}$ actually depends on the implementation.

On the other hand, Bob's computation cost in $\mathrm{PP} k \mathrm{NN}$ is mainly due to the encryption of his input query. In our dataset, Bob's computation cost is 4 and 17 milliseconds when $K$ is 512 and 1024 bits, respectively. It is apparent that $\mathrm{PP} k \mathrm{NN}$ is very efficient from Bob's computational perspective which is especially beneficial when he issues queries from a resource-constrained device (such as mobile phone and PDA).

\subsection{Communication Costs of PP $k N N$}

The communication costs of PP $k N N$ for $k=10$ and $K=1024$ bits are shown in Table 3 . Specifically, the total communication sizes of Stages 1 and 2 in $\mathrm{PP} k \mathrm{NN}$ are 154.741 and $0.037 \mathrm{MB}$, respectively. By assuming a standard $10 \mathrm{Mbps}$ LAN setting, the corresponding network delays between $C_{1}$ and $C_{2}$ are 123.79 and 0.0296 seconds, respectively. Here it is evident that the total network delay (around 2 minutes) of $\mathrm{PP} k \mathrm{NN}$ is significantly less than its total computation cost. Similar conclusions can be drawn for other parameter settings.

\section{Conclusion}

Classification is an important task in many data mining applications such as detection of fraud by credit card companies and prediction of tumor cells levels in blood. To protect user privacy, various privacy-preserving classification techniques have been proposed in the literature for the past decade. Nevertheless, the existing techniques are not applicable in outsourced database environment where the data resides in encrypted form on a third-party server. Along this direction, this paper proposed a novel privacy-preserving $k$-NN classification protocol over encrypted data in the cloud. Our protocol protects the confidentiality of the data, user's input query, and hides the data access patterns. We also evaluated the performance of our protocol under different parameter settings.

Since improving the efficiency of $\mathrm{SMIN}_{n}$ is an important first step for improving the performance of our PP $k \mathrm{NN}$ protocol, we plan to investigate alternative and more efficient solutions to the $\operatorname{SMIN}_{n}$ problem in our future work. Also, in this paper, we used the well-known $k$-NN classifier and developed a privacy-preserving protocol for it over encrypted data. As a future work, we will investigate and extend our research to other classification algorithms.

\section{References}

[1] C. C. Aggarwal and P. S. Yu. A general survey of privacy-preserving data mining models and algorithms. Privacy-preserving data mining, pages 11-52, 2008.

[2] R. Agrawal, J. Kiernan, R. Srikant, and Y. Xu. Order preserving encryption for numeric data. In ACM SIGMOD, pages 563-574, 2004. 
[3] R. Agrawal and R. Srikant. Privacy-preserving data mining. In ACM Sigmod Record, volume 29, pages 439-450. ACM, 2000.

[4] Y. Aumann and Y. Lindell. Security against covert adversaries: Efficient protocols for realistic adversaries. Journal of Cryptology, 23(2):281-343, Apr. 2010.

[5] R. J. Bayardo and R. Agrawal. Data privacy through optimal k-anonymization. In IEE ICDE, pages 217-228, 2005.

[6] D. Beaver. Foundations of secure interactive computing. In Advances in Cryptology - CRYPTO '91, pages 377-391. Springer-Verlag, 1991.

[7] A. Ben-David, N. Nisan, and B. Pinkas. Fairplaymp - a system for secure multi-party computation. In ACM CCS, October 2008.

[8] D. Bogdanov, S. Laur, and J. Willemson. Sharemind: A framework for fast privacy-preserving computations. In Proceedings of the 13th European Symposium on Research in Computer Security: Computer Security, ESORICS '08, pages 192-206. Springer, 2008.

[9] M. Bohanec and B. Zupan. The UCI KDD Archive. University of California, Department of Information and Computer Science, Irvine, CA, 1997. http://archive.ics.uci.edu/ml/datasets/Car+Evaluation.

[10] R. Buyya, C. S. Yeo, S. Venugopal, J. Broberg, and I. Brandic. Cloud computing and emerging it platforms: Vision, hype, and reality for delivering computing as the 5th utility. Future Generation computer systems, 25(6):599-616, 2009.

[11] J. Camenisch and M. Michels. Proving in zero-knowledge that a number is the product of two safe primes. In EUROCRYPT, pages 107-122. Springer-Verlag, 1999.

[12] R. Canetti. Security and composition of multiparty cryptographic protocols. Journal of Cryptology, 13(1):143$202,2000$.

[13] R. Canetti. Universally composable security: a new paradigm for cryptographic protocols. In IEEE FOCS, pages $136-145$, oct. 2001.

[14] D. Chaum, C. Crépeau, and I. Damgard. Multiparty unconditionally secure protocols. In Proceedings of the Twentieth Annual ACM Symposium on Theory of Computing, STOC '88, pages 11-19. ACM, 1988.

[15] C. Clifton, M. Kantarcioglu, J. Vaidya, X. Lin, and M. Y. Zhu. Tools for privacy preserving distributed data mining. ACM SIGKDD Explorations Newsletter, 4(2):28-34, 2002.

[16] R. Cramer, I. Damgård, and J. B. Nielsen. Multiparty computation from threshold homomorphic encryption. In Advances in Cryptology - EUROCRYPT, pages 280-299, 2001.

[17] I. Damgård and M. Jurik. A generalisation, a simplification and some applications of paillier's probabilistic public-key system. In Proceedings of the 4th International Workshop on Practice and Theory in Public Key Cryptography, pages 119-136. Springer-Verlag, 2001.

[18] I. Damgård and M. Jurik. A length-flexible threshold cryptosystem with applications. In Proceedings of the Australasian conference on Information security and privacy, pages 350-364. Springer-Verlag, 2003.

[19] S. De Capitani di Vimercati, S. Foresti, and P. Samarati. Managing and accessing data in the cloud: Privacy risks and approaches. In 7th International Conference on Risk and Security of Internet and Systems (CRiSIS), pages 1 $-9,2012$. 
[20] Y. Elmehdwi, B. K. Samanthula, and W. Jiang. Secure $k$-nearest neighbor query over encrypted data in outsourced environments. In the 30th IEEE International Conference on Data Engineering (ICDE), 2014. To appear. http://web.mst.edu/ wjiang/SkNN-ICDE14.pdf

[21] A. Evfimievski, R. Srikant, R. Agrawal, and J. Gehrke. Privacy preserving mining of association rules. Information Systems, 29(4):343-364, 2004.

[22] S. Fienberg and J. McIntyre. Data swapping: Variations on a theme by dalenius and reiss. In Privacy in statistical databases, pages 519-519. Springer, 2004.

[23] P.-A. Fouque, G. Poupard, and J. Stern. Sharing decryption in the context of voting or lotteries. In Proceedings of the 4th International Conference on Financial Cryptography, pages 90-104, 2001.

[24] C. Gentry. Fully homomorphic encryption using ideal lattices. In ACM STOC, pages 169-178, 2009.

[25] C. Gentry and S. Halevi. Implementing gentry's fully-homomorphic encryption scheme. In EUROCRYPT, pages 129-148. Springer-Verlag, 2011.

[26] O. Goldreich. The Foundations of Cryptography, volume 2, chapter Encryption Schemes, pages 373-470. Cambridge University Press, Cambridge, England, 2004.

[27] O. Goldreich. The Foundations of Cryptography, volume 2, chapter General Cryptographic Protocols, pages 599-746. Cambridge, University Press, Cambridge, England, 2004.

[28] O. Goldreich, S. Micali, and A. Wigderson. How to play any mental game - a completeness theorem for protocols with honest majority. In 19th Symposium on the Theory of Computing, pages 218-229, New York, 1987. ACM.

[29] S. Goldwasser, S. Micali, and C. Rackoff. The knowledge complexity of interactive proof systems. SIAM Journal of Computing, 18:186-208, February 1989.

[30] H. Hacigümüş, B. Iyer, C. Li, and S. Mehrotra. Executing sql over encrypted data in the database-service-provider model. In ACM SIGMOD, pages 216-227, 2002.

[31] W. Henecka, S. K ögl, A.-R. Sadeghi, T. Schneider, and I. Wehrenberg. Tasty: tool for automating secure two-party computations. In ACM CCS, pages 451-462. ACM, 2010.

[32] B. Hore, S. Mehrotra, M. Canim, and M. Kantarcioglu. Secure multidimensional range queries over outsourced data. The VLDB Journal, 21(3):333-358, 2012.

[33] H. Hu, J. Xu, C. Ren, and B. Choi. Processing private queries over untrusted data cloud through privacy homomorphism. In IEEE ICDE, pages 601-612, 2011.

[34] Y. Huang, D. Evans, and J. Katz. Private set intersection: Are garbled circuits better than custom protocols? In NDSS, 2012.

[35] Y. Huang, D. Evans, J. Katz, and L. Malka. Faster secure two-party computation using garbled circuits. In Proceedings of the 20th USENIX conference on Security (SEC'11), pages 35-35, 2011.

[36] Y. Huang, J. Katz, and D. Evans. Quid-pro-quo-tocols: Strengthening semi-honest protocols with dual execution. In IEEE Symposium on Security and Privacy, pages 272-284. IEEE Computer Society, 2012.

[37] M. Kantarcioglu and C. Clifton. Privately computing a distributed k-nn classifier. In Proceedings of the 8th European Conference on Principles and Practice of Knowledge Discovery in Databases, PKDD '04, pages 279290, New York, NY, USA, 2004. Springer-Verlag.

[38] J. Katz and Y. Lindell. Introduction to Modern Cryptography. Chapman \& Hall, CRC Press, 2007.

[39] Y. Lindell. General composition and universal composability in secure multiparty computation. Journal of Cryptology, 22(3):395-428, 2009. 
[40] Y. Lindell and B. Pinkas. Privacy preserving data mining. In Advances in Cryptology (CRYPTO), pages 36-54. Springer, 2000.

[41] Y. Lindell and B. Pinkas. Secure multiparty computation for privacy-preserving data mining. Journal of Privacy and Confidentiality, 1(1):5, 2009.

[42] P. Mell and T. Grance. The nist definition of cloud computing (draft). NIST special publication, 800:145, 2011.

[43] V. Nikolaenko, U. Weinsberg, S. Ioannidis, M. Joye, D. Boneh, and N. Taft. Privacy-preserving ridge regression on hundreds of millions of records. In IEEE Symposium on Security and Privacy (SP '13), pages 334-348. IEEE Computer Society, 2013.

[44] S. R. Oliveira and O. R. Zaiane. Privacy preserving clustering by data transformation. In Proc. of the 18th Brazilian Symposium on Databases, pages 304-318, 2003.

[45] P. Paillier. Public key cryptosystems based on composite degree residuosity classes. In Eurocrypt, pages 223238. Springer-Verlag, 1999.

[46] S. Pearson and A. Benameur. Privacy, security and trust issues arising from cloud computing. In IEEE CloudCom, pages 693-702, 2010.

[47] Y. Qi and M. J. Atallah. Efficient privacy-preserving k-nearest neighbor search. In Proceedings of the 28th International Conference on Distributed Computing Systems, pages 311-319, Washington, DC, USA, 2008. IEEE Computer Society.

[48] S. Ravu, P. Neelakandan, M. Gorai, R. Mukkamala, and P. Baruah. A computationally efficient and scalable approach for privacy preserving knn classification. In IEEE International Conference on High Performance Computing (HiPC), 2012.

[49] A. Sahai. Computing on encrypted data. Information Systems Security, pages 148-153, 2008.

[50] B. K. Samanthula and W. Jiang. An efficient and probabilistic secure bit-decomposition. In 8th ACM Symposium on Information, Computer and Communications Security (ASIACCS), pages 541-546, 2013.

[51] A. Shamir. How to share a secret. Commun. ACM, 22(11):612-613, Nov. 1979.

[52] P. Williams, R. Sion, and B. Carbunar. Building castles out of mud: practical access pattern privacy and correctness on untrusted storage. In ACM CCS, pages 139-148, 2008.

[53] W. K. Wong, D. W.-l. Cheung, B. Kao, and N. Mamoulis. Secure knn computation on encrypted databases. In ACM SIGMOD, pages 139-152, 2009.

[54] X. Xiao, F. Li, and B. Yao. Secure nearest neighbor revisited. In IEEE ICDE, pages 733-744, 2013.

[55] L. Xiong, S. Chitti, and L. Liu. K nearest neighbor classification across multiple private databases. In Proceedings of the 15th ACM International Conference on Information and Knowledge Management, pages 840-841, New York, NY, USA, 2006. ACM.

[56] A. C. Yao. Protocols for secure computations. In Proceedings of the 23rd Annual Symposium on Foundations of Computer Science, pages 160-164, Washington, DC, USA, 1982. IEEE Computer Society.

[57] A. C. Yao. How to generate and exchange secrets. In Proceedings of the 27th Symposium on Foundations of Computer Science, pages 162-167, Washington, DC, USA, 1986. IEEE Computer Society.

[58] P. Zhang, Y. Tong, S. Tang, and D. Yang. Privacy preserving naive bayes classification. Advanced Data Mining and Applications, pages 730-730, 2005. 